\title{
Comprehensive Numerical Classification of 369 Strains of Mycobacterium, Rhodococcus, and Nocardia
}

\author{
MICHIO TSUKAMURA, ' SHOJI MIZUNO,' SUMIO TSUKAMURA, ${ }^{2}$ AND JUNKO TSUKAMURA ${ }^{2}$ \\ The National Chubu Hospital, Obu, ${ }^{1}$ and Tsukamura Clinic, Obu (Kyowa), ${ }^{2}$ Aichi 474, Japan
}

\begin{abstract}
A numerical analysis of 369 strains received as members of the genus $M y c o-$ bacterium, Rhodococcus (formerly Gordona), Nocardia, or Corynebacterium was carried out using 88 characters. Of the strains tested, 345 mycobacteria, rhodococci, and nocardiae formed four distinct clusters at a similarity level of $85 \%$. The first cluster was composed of slowly growing mycobacteria; the second cluster, of strains of Mycobacterium flavescens and $M$. thermoresistibile; the third cluster, of strains of rapidly growing mycobacteria; and the fourth cluster, of strains of Rhodococcus and Nocardia. Strains of three species of rapidly growing mycobacteria, $M$. agri, $M$. smegmatis, and $M$. vaccae, were located outside of these clusters. The fourth cluster could be divided into three subclusters, one consisting of rhodococci plus some strains of Nocardia asteroides, a second consisting of the remaining strains of Nocardia, and a third consisting of strains of Rhodococcus aurantiacus (Gordona aurantiaca) and Jensenia canicruria.
\end{abstract}

Mycobacterium, Rhodococcus (formerly Gor. dona), and Nocardia are closely related taxa belonging to the order Actinomycetales (21).

In 1966, Tsukamura (13) reported that mycobacteria can be divided into two subgroups, which roughly correspond to slowly growing and rapidly growing mycobacteria, by numerical classification. These two subgroups differed from each other not only in growth rate, but also in several biological characters. Subsequently, Tsukamura and Mizuno $(14,24)$ proposed the subgeneric names Mycobacterium and Mycomycobacterium for these subgroups. Later, after having compared mycobacteria and nocardiae, Tsukamura (17) recommended that the subgenera Mycobacterium (slowly growing mycobacteria) and Mycomycobacterium (rapidly growing mycobacteria) be elevated to genus status, i.e., taxa equivalent in status to the genus Nocardia.

In 1971, Tsukamura (18) proposed a new genus, Gordona, for slightly acid-fast organisms considered to be intermediate between $\mathrm{Myco}$ bacterium and Nocardia and placed it in the family Actinomycetaceae, to which the genus Nocardia belongs. It was later shown by Tsukamura that the genus Gordona contained strain ATCC 13808 of Rhodococcus rhodochrous (19), and it was stated that the generic name Gordona should be changed to Rhodococcus (20), as this is the first name used for an organism belonging to the genus proposed. Independently, Goodfellow (2) observed that ' $M y$ cobacterium" rhodochrous differs from nocardiae, and Goodfellow et al. (4) reported that it also differs from rapidly growing mycobacteria.
The finding that ' $M$." rhodochrous differs from both Mycobacterium and Nocardia was confirmed by an international cooperative study (5). Goodfellow and Alderson (3) also proposed the name Rhodococcus for the "M." rhodochrous complex, in which they included the genus Gordona. These authors also included several new species in the genus Rhodococcus.

The taxonomic relationships of the mycobacteria, rhodococci, and nocardiae were studied by Tsukamura (21), who included hypothetical median organisms of representative species of these taxa in his study. Tsukamura proposed that the slowly growing mycobacteria, the rapidly growing mycobacteria, Gordona (now Rhodococcus), and Nocardia be regarded as distinct taxa. He noted that the slowly growing mycobacteria seemed to be only distantly related to the other three groups, which showed a close relationship with each other.

The purpose of the present study was to conduct a numerical taxonomy analysis of a large number of strains belonging to these taxa. A total of 369 strains of Mycobacterium, Rhodococcus, and Nocardia and 4 strains of Corynebacterium were investigated.

\section{MATERIALS AND METHODS}

Strains. A total of 365 strains received as members of Mycobacterium, Rhodococcus, or Nocardia and 4 strains received as members of Corynebacterium were studied (see Table 1).

Tests. A total of 88 characters were used in the numerical analysis. These characters were selected from 118 characters routinely determined in this 
TABLE 1. Strains used in this study

\begin{tabular}{|c|c|c|c|c|c|}
\hline \multirow{2}{*}{$\begin{array}{c}\text { Serial } \\
\text { no. }\end{array}$} & \multirow{2}{*}{$\begin{array}{l}\text { Labora- } \\
\text { tory no. }\end{array}$} & \multicolumn{2}{|c|}{ Received as: } & \multirow{2}{*}{ Source ${ }^{a}$} & \multirow{2}{*}{ Habitat } \\
\hline & & Name & Strain & & \\
\hline 1 & $\mathrm{E} 6383$ & Mycobacterium tuberculosis & TC1 & (1) & Humans \\
\hline 2 & E6384 & Mycobacterium tuberculosis & TC5 & (1) & Humans \\
\hline 3 & E6543 & Mycobacterium tuberculosis & TC32 & (1) & Humans \\
\hline 4 & E6548 & Mycobacterium tuberculosis & TC40 & (1) & Humans \\
\hline 5 & $05001 B$ & Mycobacterium tuberculosis & H37Rv & (2) & Humans \\
\hline 6 & 05002 & Mycobacterium tuberculosis & Aoyama-B & (2) & Humans \\
\hline 7 & E6542 & Mycobacterium tuberculosis & TC22 & (1) & Humans \\
\hline 8 & E7949 & Mycobacterium tuberculosis & & (3) & Sputa, humans \\
\hline 9 & E6165 & Mycobacterium tuberculosis & & (3) & Sputa, humans \\
\hline 10 & E6385 & Mycobacterium tuberculosis & TC6 & (1) & Humans \\
\hline 11 & E6387 & Mycobacterium tuberculosis & TC9 & (1) & Humans \\
\hline 12 & E6401 & Mycobacterium tuberculosis & TC29 & (1) & Humans \\
\hline 13 & E6661 & Mycobacterium tuberculosis & TC13 & (1) & Humans \\
\hline 14 & E6662 & Mycobacterium tuberculosis & TC15 & (1) & Humans \\
\hline 15 & E7193 & Mycobacterium tuberculosis & & (3) & Sputa, humans \\
\hline 16 & E7966 & Mycobacterium tuberculosis & & (3) & Sputa, humans \\
\hline 17 & E7950 & Mycobacterium tuberculosis & & (3) & Sputa, humans \\
\hline 18 & $05001 \mathrm{~A}$ & Mycobacterium tuberculosis & H37Rv & (2) & Humans \\
\hline 19 & E6394 & Mycobacterium tuberculosis & $\mathrm{TC} 21$ & (1) & Humans \\
\hline $\begin{array}{l}19 \\
20\end{array}$ & $\begin{array}{l}\text { L0034 } \\
\text { E6549 }\end{array}$ & Mycobacterium africanum & TC41 & (1) & Humans \\
\hline 21 & E6677 & Mycobacterium microti & TC62 & (1) & $?$ \\
\hline 22 & E6567 & Mycobacterium africanum & TC93 & (1) & Humans \\
\hline 23 & E6689 & Mycobacterium africanum & TC84 & (1) & Humans \\
\hline 24 & E6386 & Mycobacterium bovis & TC7 & (1) & Milk \\
\hline 25 & E6537 & Mycobacterium africanum & TC3 & (1) & Humans \\
\hline 26 & E6412 & Mycobacterium bovis & TC92 & (1) & Dogs \\
\hline 27 & E6558 & Mycobacterium africanum & TC67 & (1) & Humans \\
\hline 28 & E6565 & Mycobacterium africanum & TC88 & (1) & Humans \\
\hline 29 & E6683 & Mycobacterium africanum & TC74 & (1) & Humans \\
\hline 30 & E6544 & Mycobacterium bovis & TC34 & (1) & Cows \\
\hline 31 & E6554 & Mycobacterium africanum & TC57 & (1) & Humans \\
\hline 32 & E6560 & Mycobacterium africanum & TC73 & (1) & Humans \\
\hline 33 & E6664 & Mycobacterium africanum & TC37 & (1) & Humans \\
\hline $\begin{array}{l}30 \\
34\end{array}$ & E60078 & Mycobacterium bovis & TC65 & (1) & Deer \\
\hline $\begin{array}{l}34 \\
35\end{array}$ & E6692 & Mycobacterium africanum & TC8 & (1) & Humans \\
\hline $\begin{array}{l}00 \\
36\end{array}$ & E6393 & Mycobacterium africanum & TC20 & (1) & Humans \\
\hline 37 & E6396 & Mycobacterium bovis & TC24 & (1) & Cows \\
\hline 38 & E6671 & Mycobacterium bovis & TC51 & (1) & Bison \\
\hline 39 & E6402 & Mycobacterium bovis & TC30 & (1) & Cows \\
\hline 40 & $06001 \mathrm{~A}$ & Mycobacterium bovis & Ravenel & (4) & Cows \\
\hline 41 & 06002 & Mycobacterium bovis & Denken & (5) & Cows \\
\hline $\begin{array}{l}41 \\
42\end{array}$ & $06001 B$ & Mycobacterium bovis & Ravenel & (4) & Cows \\
\hline $\begin{array}{l}42 \\
43\end{array}$ & E6675 & Mycobacterium bovis & TC58 & (1) & Cows \\
\hline $\begin{array}{l}43 \\
44\end{array}$ & E6388 & Mycobacterium bovis & TC11 & (1) & BCG (Czech strain) \\
\hline 45 & E6691 & Mycobacterium microti & TC89 & (1) & Llamas \\
\hline 46 & E6392 & Mycobacterium microti & TC19 & (1) & $?$ \\
\hline 47 & E6398 & Mycobacterium microti & TC26 & (1) & Voles \\
\hline 48 & E6400 & Mycobacterium microti & $\mathrm{TC28}$ & (1) & $?$ \\
\hline 49 & E6668 & Mycobacterium microti & TC48 & (1) & Cats \\
\hline 50 & E6688 & Mycobacterium bovis & TC83 & (1) & Minks \\
\hline 51 & E7743 & Mycobacterium intracellulare & 13444 & (6) & Humans \\
\hline 52 & E7744 & Mycobacterium intracellulare & 18730 & (6) & Humans \\
\hline 53 & E7747 & Mycobacterium intracellulare & 25356 & (6) & Humans \\
\hline 54 & E7748 & Mycobacterium intracellulare & 28659 & (6) & Sewage \\
\hline $\begin{array}{l}04 \\
55\end{array}$ & E7741 & Mycobacterium intracellulare & 4130 & (6) & Humans \\
\hline $\begin{array}{l}00 \\
56\end{array}$ & E7745 & & 23774 & (6) & Humans \\
\hline $\begin{array}{l}50 \\
57\end{array}$ & $\begin{array}{l}\text { Lil } \\
\text { E7749 }\end{array}$ & Mycobacterium intracellulare & 28660 & (6) & $?$ \\
\hline $\begin{array}{l}5 \\
58\end{array}$ & E7750 & Mycobacterium intracellulare & 28661 & (6) & Birds \\
\hline $\begin{array}{l}50 \\
59\end{array}$ & E7742 & Mycobacterium intracellulare & $\begin{array}{l}20001 \\
10499\end{array}$ & (6) & Humans \\
\hline 60 & E7746 & Mycobacterium intracellulare & 24657 & (6) & Humans \\
\hline
\end{tabular}


TABLE 1 (continued)

\begin{tabular}{|c|c|c|c|c|c|}
\hline \multirow{2}{*}{$\begin{array}{c}\text { Serial } \\
\text { no. }\end{array}$} & \multirow{2}{*}{$\begin{array}{l}\text { Labora- } \\
\text { tory no. }\end{array}$} & \multicolumn{2}{|l|}{ Received as: } & \multirow{2}{*}{ Source $^{a}$} & \multirow{2}{*}{ Habitat } \\
\hline & & Name & Strain & & \\
\hline 61 & 13022 & Mycobacterium intracellulare & NJ-16 & (3) & Humans \\
\hline 62 & 13039 & Mycobacterium intracellulare & NJ-17 & (3) & Humans \\
\hline 63 & E6111 & Mycobacterium intracellulare & & (3) & Sputa, humans \\
\hline 64 & E6172 & Mycobacterium intracellulare & & (3) & Sputa, humans \\
\hline 65 & 13014 & Mycobacterium intracellulare & & (3) & Sputa, humans \\
\hline 66 & 13015 & Mycobacterium intracellulare & & (3) & Sputa, humans \\
\hline 67 & 13023 & Mycobacterium intracellulare & NJ-7 & (3) & Sputa, man \\
\hline 68 & E7959 & Mycobacterium intracellulare & TS-P & (7) & Swine, lymph nodes \\
\hline 69 & E7961 & Mycobacterium intracellulare & No. 23 & (7) & Swine, lymph nodes \\
\hline 70 & 13024 & Mycobacterium intracellulare & NJ-8 & (3) & Humans \\
\hline 71 & 13032 & Mycobacterium intracellulare & $\mathrm{NJ}-10$ & (3) & Humans \\
\hline 72 & 13038 & Mycobacterium intracellulare & $\mathrm{NJ}-6$ & (3) & Humans \\
\hline 73 & 13936 & Mycobacterium intracellulare & & (3) & Humans \\
\hline 74 & E7957 & Mycobacterium intracellulare & TS-H & (7) & Swine, lymph nodes \\
\hline 75 & E7963 & Mycobacterium intracellulare & & (3) & Sputa, humans \\
\hline 76 & 13543 & Mycobacterium intracellulare & ATCC 19177 & (8) & Hens \\
\hline 77 & E7958 & Mycobacterium intracellulare & TS-L & (7) & Swine \\
\hline 78 & 11016 & Mycobacterium avium & ATCC 15769 & (8) & Hens \\
\hline 79 & 11021 & Mycobacterium avium & ATCC 17942 & (8) & $?$ \\
\hline 80 & 11018 & Mycobacterium avium & ATCC 17941 & (8) & $?$ \\
\hline 81 & 11017 & Mycobacterium avium & ATCC 19075 & (8) & Humans, lymph nodes \\
\hline 82 & 11019 & Mycobacterium avium & ATCC 17944 & (8) & ? \\
\hline 83 & 11020 & Mycobacterium avium & ATCC 15773 & (8) & ? \\
\hline 84 & E7682 & Mycobacterium intracellulare & & (3) & Sputa, humans \\
\hline 85 & E7954 & Mycobacterium intracellulare & A-1 & (7) & Swine, lymph nodes \\
\hline 86 & 11025 & Mycobacterium avium? & ATCC 17940 & (8) & $?$ \\
\hline 87 & E7960 & Mycobacterium intracellulare & No. 6 & (7) & Swine, lymph nodes \\
\hline 88 & 13013 & Mycobacterium intracellulare & NJ-1 & (3) & Humans \\
\hline 89 & 13016 & Mycobacterium intracellulare & NJ-2 & (3) & Humans \\
\hline 90 & 13019 & Mycobacterium intracellulare & NJ-18 & (3) & Humans \\
\hline 91 & 13542 & ? & ATCC 19076 & (8) & Humans \\
\hline 92 & 13935 & Mycobacterium intracellulare & & (3) & Sputa, humans \\
\hline 93 & 12004 & Mycobacterium scrofulaceum & SJ-8 & (3) & Humans \\
\hline 94 & 12008 & Mycobacterium scrofulaceum & SJ-9 & (3) & Humans \\
\hline 95 & 12014 & Mycobacterium scrofulaceum & SJ-1 & (3) & Humans \\
\hline 96 & $12007 \mathrm{~A}$ & Mycobacterium scrofulaceum & SJ-5 & (3) & Humans \\
\hline 97 & 12017 & Mycobacterium scrofulaceum & SJ-12 & (3) & Humans \\
\hline 98 & $12007 \mathrm{~B}$ & Mycobacterium scrofulaceum & SJ-5 & (3) & Humans \\
\hline 99 & $12016 \mathrm{~A}$ & Mycobacterium scrofulaceum & SJ-14 & (3) & Humans \\
\hline 100 & 12012 & Mycobacterium scrofulaceum & SJ-3 & (3) & Humans \\
\hline 101 & 12019 & Mycobacterium scrofulaceum & SJ-15 & (3) & Humans \\
\hline 102 & 12002 & Mycobacterium scrofulaceum & P-6 & (9) & Humans \\
\hline 103 & 12009 & Mycobacterium scrofulaceum & SJ-13 & (3) & Humans \\
\hline 104 & 12020 & Mycobacterium scrofulaceum & SJ-16 & (3) & Humans \\
\hline 105 & 12321 & Mycobacterium scrofulaceum & ATCC 15078 & (8) & $?$ \\
\hline 106 & 12320 & Mycobacterium scrofulaceum & ATCC 19981 (type) & (8) & Cervical lymph nodes \\
\hline 107 & 12107 & Mycobacterium scrofulaceum & ATCC 15978 & (8) & $?$ \\
\hline 108 & 12108 & Mycobacterium scrofulaceum & ATCC 19073 & (8) & Sputa, humans \\
\hline 109 & E7814 & Mycobacterium scrofulaceum & & (3) & Sputa, humans \\
\hline 110 & 13009 & Mycobacterium intracellulare & NJ-9 & (3) & Humans \\
\hline 111 & 13011 & Mycobacterium intracellulare & NJ-3 & (3) & Humans \\
\hline 112 & 36001 & $\begin{array}{l}\text { Mycobacterium scrofulaceum } \\
\text { (marianum) }\end{array}$ & ATCC 19275 & (8) & Humans \\
\hline 113 & 12178 & Mycobacterium gordonae & ATCC 23284 & (8) & $?$ \\
\hline 114 & E6009 & Mycobacterium intracellulare? & & (3) & Humans \\
\hline 115 & E6541 & Mycobacterium bovis & TC18 & (1) & $?$ \\
\hline 116 & 12001 & Mycobacterium scrofulaceum & P-5 & (9) & Humans \\
\hline 117 & 12109 & Mycobacterium gordonae & ATCC 14470 (type) & (8) & Gastric lavage \\
\hline 118 & 12110 & Mycobacterium gordonae & ATCC 19277 & (8) & Tap water \\
\hline 119 & $12016 \mathrm{~B}$ & Mycobacterium gordonae & SJ-6 & (3) & Humans \\
\hline
\end{tabular}


TABLE 1 (continued)

\begin{tabular}{|c|c|c|c|c|c|}
\hline \multirow{2}{*}{$\begin{array}{l}\text { Serial } \\
\text { no. }\end{array}$} & \multirow{2}{*}{$\begin{array}{l}\text { Labora- } \\
\text { tory no. }\end{array}$} & \multicolumn{2}{|c|}{ Received as: } & \multirow{2}{*}{ Source" } & \multirow{2}{*}{ Habitat } \\
\hline & & Name & Strain & & \\
\hline 120 & 12177 & Mycobacterium gordonae & ATCC 23283 & (8) & $?$ \\
\hline 121 & 12180 & Mycobacterium gordonae & ATCC 23286 & (8) & Sputa, humans \\
\hline 122 & 12179 & Mycobacterium gordonae & ATCC 23285 & (8) & Tap water \\
\hline 123 & 44008 & Mycobacterium gordonae? & S-25-2 & (7) & Cows, thelitis lesions \\
\hline 124 & 44009 & Mycobacterium gordonae? & S-25-2B & (7) & Cows, thelitis lesions \\
\hline 125 & 44007 & Mycobacterium gordonae? & $0-25-2$ & (7) & Cows, thelitis lesions \\
\hline 126 & E6117 & Mycobacterium intracellulare & & (3) & Sputa, humans \\
\hline 127 & E6110 & Mycobacterium intracellulare & & (3) & Sputa, humans \\
\hline 128 & 28001 & Mycobacterium xenopi & ATCC 19156 & (8) & Humans \\
\hline 129 & 28002 & Mycobacterium xenopi & ATCC 19276 & (8) & Humans \\
\hline 130 & 28003 & Mycobacterium xenopi & ATCC 19250 (type) & (8) & Adult female toads \\
\hline 131 & 28004 & Mycobacterium xenopi & ATCC 19970 & (8) & $?$ \\
\hline 132 & E6137 & Mycobacterium gordonae? & & (3) & Sputa, humans \\
\hline 133 & E7962 & $?$ & Ishikari-31 & (7) & Swine \\
\hline 134 & 09023 & $\begin{array}{l}\text { Mycobacterium nonchromo- } \\
\text { genicum }\end{array}$ & ATCC 19532 & (8) & Soil \\
\hline 135 & 09033 & $\begin{array}{l}\text { Mycobacterium nonchromo- } \\
\text { genicum }\end{array}$ & ATCC 19533 & (8) & Soil \\
\hline 136 & 09003 & $\begin{array}{l}\text { Mycobacterium nonchromo. } \\
\text { genicum }\end{array}$ & ATCC 19530 (type) & (8) & Soil \\
\hline 137 & E4322 & Mycobacterium gastri ${ }^{* b}$ & ATCC 25028 & (8) & $?$ \\
\hline 138 & E4324 & Mycobacterium gastri ${ }^{*}$ & ATCC 25030 & (8) & $?$ \\
\hline 139 & E4323 & Mycobacterium gastri* & ATCC 25029 & (8) & $?$ \\
\hline 140 & E4325 & Mycobacterium gastri* & ATCC 25031 & (8) & $?$ \\
\hline 141 & E4321 & Mycobacterium gastri* & ATCC 25027 & (8) & $?$ \\
\hline 142 & E4326 & Mycobacterium gastri* & ATCC 25032 & (8) & $?$ \\
\hline 143 & E4327 & Mycobacterium gastri* & ATCC 25033 & (8) & $?$ \\
\hline 144 & E4335 & $?$ & ATCC 25041 & (8) & $?$ \\
\hline 145 & E4342 & Mycobacterium kansasii & ATCC 25048 & (8) & $?$ \\
\hline 146 & $\mathrm{E} 4336$ & Mycobacterium kansasiï & ATCC 25042 & (8) & $?$ \\
\hline 147 & E4337 & Mycobacterium kansasii & ATCC 25043 & (8) & $?$ \\
\hline 148 & E4339 & Mycobacterium kansasii* & ATCC 25045 & (8) & $?$ \\
\hline 149 & E4340 & Mycobacterium kansasii & ATCC 25046 & (8) & $?$ \\
\hline 150 & E4341 & Mycobacterium kansasii & ATCC 25047 & (8) & $?$ \\
\hline 151 & $\mathrm{E} 4343$ & Mycobacterium kansasii ${ }^{*}$ & ATCO 25049 & (8) & $?$ \\
\hline 152 & 07004 & Mycobacterium kansasii* & Nagai & (3) & Sputa, humans \\
\hline 153 & 07012 & Mycobacterium kansasii & Chinen & (3) & Sputa, humans \\
\hline 154 & E6184 & Mycobacterium szulgai & & (3) & Sputa, humans \\
\hline 155 & $\mathrm{Z} 12001$ & Mycobacterium szulgai & 23428 & (6) & Humans \\
\hline 156 & $\mathrm{Z} 12002$ & Mycobacterium szulgai & 25932 & (6) & Humans \\
\hline 157 & $\mathrm{Z12003}$ & Mycobacterium szulgai & 26701 & (6) & Humans \\
\hline 158 & Z12004 & Mycobacterium szulgai & 20886 & (6) & Humans \\
\hline 159 & E6139 & ? & & (3) & Sputa, humans \\
\hline 160 & E4796 & Mycobacterium shimoidei & ATCC 27962 (type) & (8) & Sputa, humans \\
\hline 161 & E7380 & Mycobacterium shimoidei & & (3) & Sputa, humans \\
\hline 162 & 44006 & ? & L-25-2A & (7) & Cows, thelitis lesions \\
\hline 163 & E4328 & Mycobacterium marinum* & ATCC 25034 & (8) & $?$ \\
\hline 164 & 08001 & Mycobacterium marinum & ATCC 927 & (8) & Fish \\
\hline 165 & E6104 & ? & & (3) & Sputa, humans \\
\hline 166 & E6107 & $?$ & & (3) & Sputa, humans \\
\hline 167 & E6152 & $?$ & & (3) & Sputa, humans \\
\hline 168 & 33004 & Mycobacterium flavescens & E3364 & (3) & Sputa, humans \\
\hline 169 & 33005 & Mycobacterium flavescens & E3365 & (3) & Sputa, humans \\
\hline 170 & 33001 & Mycobacterium flavescens & ATCC 14474 (type) & (8) & Guinea pigs \\
\hline 171 & 01002 & $\begin{array}{l}\text { Mycobacterium thermoresisti- } \\
\text { bile }\end{array}$ & ATCC 19528 & (8) & Soil \\
\hline 172 & 01021 & $\begin{array}{l}\text { Mycobacterium thermoresisti- } \\
\text { bile }\end{array}$ & & (3) & Soil \\
\hline 173 & 01028 & $\begin{array}{l}\text { Mycobacterium thermoresisti- } \\
\text { bile }\end{array}$ & ATCC 19529 & (8) & Soil \\
\hline
\end{tabular}


TABle 1 (continued)

\begin{tabular}{|c|c|c|c|c|c|}
\hline \multirow{2}{*}{$\begin{array}{c}\text { Serial } \\
\text { no. }\end{array}$} & \multirow{2}{*}{$\begin{array}{l}\text { Labora- } \\
\text { tory no. }\end{array}$} & \multicolumn{2}{|c|}{ Received as: } & \multirow{2}{*}{ Source $^{a}$} & \multirow{2}{*}{ Habitat } \\
\hline & & Name & Strain & & \\
\hline 174 & 01039 & $\begin{array}{l}\text { Mycobacterium thermoresisti- } \\
\text { bile }\end{array}$ & ATCC 19527 (type) & (8) & Soil \\
\hline 175 & 01011 & $\begin{array}{l}\text { Mycobacterium thermoresisti- } \\
\text { biie }\end{array}$ & & (3) & Soil \\
\hline 176 & E5750 & Mycobacterium fortuitum & & (3) & Sputa, humans \\
\hline 177 & E6029 & Mycobacterium fortuitum & & (3) & Sputa, humans \\
\hline 178 & $\mathbf{E} 6051$ & Mycobacterium fortuitum & & (3) & Sputa, humans \\
\hline 179 & $\mathrm{E} 6035$ & Mycobacterium fortuitum & & (3) & Sputa, humans \\
\hline 180 & 18007 & Mycobacterium fortuitum & E308 & (3) & Soil \\
\hline 181 & 18110 & Mycobacterium peregrinum & ATCC 14467 (type) & (8) & Humans \\
\hline 182 & 18009 & Mycobacterium fortuitum & NCTC 8697 & (9) & $?$ \\
\hline 183 & 18001 & Mycobacterium fortuitum & ATCC 19709 & (8) & Soil \\
\hline 184 & 18010 & Mycobacterium fortuitum & R389 & (10) & $?$ \\
\hline 185 & 18011 & Mycobacterium fortuitum & E1701 & (3) & Soil \\
\hline 186 & 18109 & Mycobacterium fortuitum & ATCC 6842 & (8) & $\begin{array}{l}\text { Humans, cold ab- } \\
\text { scesses }\end{array}$ \\
\hline 187 & 10001 & Mycobacterium fortuitum & E328 & (3) & Soil \\
\hline 188 & 18112 & Mycobacterium fortuitum & ATCC 6841 (type) & (8) & $\begin{array}{l}\text { Humans, cold ab- } \\
\text { scesses }\end{array}$ \\
\hline 189 & 42001 & $\begin{array}{l}\text { Mycobacterium fortuitum (mi. } \\
\text { netti) }\end{array}$ & ATCC 19542 & (8) & $?$ \\
\hline 190 & 18021 & Mycobacterium fortuitum & E1261 & (3) & Soil \\
\hline 191 & 18024 & Mycobacterium fortuitum & E1264 & (3) & Soil \\
\hline 192 & 18022 & Mycobacterium fortuitum & E1262 & (3) & Soil \\
\hline 193 & E7820 & $?$ & N10596 & (10) & $?$ \\
\hline 194 & 15502 & Mycobacterium aurum & ATCC 25801 & (8) & Soil \\
\hline 195 & 15503 & Mycobacterium aurum & ATCC 25802 & (8) & Soil \\
\hline 196 & 22011 & Mycobacterium abscessus ${ }^{c}$ & ATCC 14472 & (8) & $?$ \\
\hline 197 & 22019 & Mycobacterium abscessus ${ }^{c}$ & ATCC 23045 & (8) & $\dot{?}$ \\
\hline 198 & 22021 & Mycobacterium abscessus ${ }^{c}$ & E2846 & (3) & Humans, lungs \\
\hline 199 & 22012 & Mycobacterium abscessus ${ }^{c}$ & ATCC 19977 (type) & (8) & Abscesses, humans \\
\hline 200 & 22016 & Mycobacterium abscessus ${ }^{c}$ & ATCC 23018 & (8) & ? \\
\hline 201 & E5765 & Mycobacterium abscessus ${ }^{c}$ & & (3) & Sputa, humans \\
\hline 202 & 19009 & Mycobacterium chelonei ${ }^{d}$ & ATCC 19235 & (8) & Gastric lavage \\
\hline 203 & 19010 & Mycobacterium chelone ${ }^{-d}$ & ATCC 19237 & (8) & Gastric lavage \\
\hline 204 & 19040 & Mycobacterium cheloneid & ATCC 23000 & (8) & $?$ \\
\hline 205 & 19041 & Mycobacterium cheloneid & ATCC 23013 & (8) & $\dot{?}$ \\
\hline 206 & 19043 & Mycobacterium chelonei ${ }^{d}$ & ATCC 23030 & (8) & $\dot{?}$ \\
\hline 207 & E7818 & ? & N34 & (11) & ? \\
\hline 208 & 14003 & Mycobacterium phlei & SN103 & (12) & ? \\
\hline 209 & 14017 & Mycobacterium phlei & Trudeau & (13) & $?$ \\
\hline 210 & 14002 & Mycobacterium phlei & SN102 & (12) & $?$ \\
\hline 211 & 14004 & Mycobacterium phlei & SN104 & (12) & $?$ \\
\hline 212 & 14022 & Mycobacterium phlei & ATCC 19249 & (8) & $?$ \\
\hline 213 & 16001 & Mycobacterium parafortuitum & ATCC 19687 & (8) & Soil \\
\hline 214 & 16002 & Mycobacterium parafortuitum & ATCC 19686 (type) & (8) & Soil \\
\hline 215 & 16003 & Mycobacterium parafortuitum & ATCC 19688 & (8) & Soil \\
\hline 216 & 48008 & Mycobacterium chubuense & E5511 & (3) & Soil \\
\hline 217 & 48012 & Mycobacterium chubuense & ATCC 27279 & (8) & Soil \\
\hline 218 & 48013 & Mycobacterium chubuense & ATCC 27278 (type) & (8) & Soil \\
\hline 219 & 49004 & Mycobacterium aichiense & ATCC 27281 & (8) & Soil \\
\hline 220 & 49005 & Mycobacterium aichiense & ATCC 27280 (type) & (8) & Soil \\
\hline 221 & 49007 & Mycobacterium aichiense & E5547 & (3) & Soil \\
\hline 222 & E5795 & Mycobacterium rhodesiae & ATCC 27024 (type) & (8) & Sputa, humans \\
\hline 223 & 31002 & Mycobacterium chitae & ATCC 19627 (type) & (8) & Soil \\
\hline 224 & 31003 & Mycobacterium chitae & ATCC 19628 & (8) & Soil \\
\hline 225 & 31004 & Mycobacterium chitae & ATCC 19629 & (8) & Soil \\
\hline 226 & E7566 & Nocardia rubra & $\mathrm{N} 20$ & (14) & $?$ \\
\hline 227 & E7568 & Nocardia rubra & N24 & (14) & ? \\
\hline 228 & E7567 & Nocardia rubra & N21 & (14) & ? \\
\hline
\end{tabular}


TABLE 1 (continued)

\begin{tabular}{|c|c|c|c|c|c|}
\hline \multirow{2}{*}{$\begin{array}{c}\text { Serial } \\
\text { no. }\end{array}$} & \multirow{2}{*}{$\begin{array}{l}\text { Labora- } \\
\text { tory no. }\end{array}$} & \multicolumn{2}{|l|}{ Received as: } & \multirow{2}{*}{ Source ${ }^{a}$} & \multirow{2}{*}{ Habitat } \\
\hline & & Name & Strain & & \\
\hline 229 & E7572 & Nocardia rubra & N50 & (14) & $?$ \\
\hline 230 & E7570 & Nocardia rubra & N26 & (14) & $?$ \\
\hline 231 & 23002 & Rhodococcus lentifragmentus & M-1 (type) & (15) & $?$ \\
\hline 232 & 23022 & Rhodococcus lentifragmentus & M-103 & (15) & $?$ \\
\hline 233 & 23022 & Rhodococcus lentifragmentus & $\mathrm{M}-122$ & (15) & $?$ \\
\hline 234 & 23016 & Rhodococcus lentifragmentus & C-1 & (15) & $?$ \\
\hline 235 & 23024 & Rhodococcus lentifragmentus & M-192 & (15) & $?$ \\
\hline 236 & E7573 & Nocardia rubra & N51 & (14) & $?$ \\
\hline 237 & 40017 & Rhodococcus rhodochrous & ATCC 4273 & (8) & ? \\
\hline 238 & 40020 & Rhodococcus rhodochrous & ATCC 14347 & (8) & ? \\
\hline 239 & 40021 & Rhodococcus rhodochrous & ATCC 14348 & (8) & $?$ \\
\hline 240 & 40022 & Rhodococcus rhodochrous & ATCC 14349 & (8) & $?$ \\
\hline 241 & E3621 & Rhodococccus terrae & & (3) & Soil \\
\hline 242 & E3622 & Rhodococcus terrae & & (3) & Soil \\
\hline 243 & E3612 & Rhodococcus terrae & & (3) & Soil \\
\hline 244 & 70006 & Rhodococcus terrae & ATCC 25594 (type) & (8) & Soil \\
\hline 245 & E5441 & Rhodococcus rubropertinctus ${ }^{h}$ & & (3) & Soil \\
\hline 246 & 60016 & Rhodococcus rubropertinctus ${ }^{h}$ & ATCC 14343 (type) & (8) & Soil \\
\hline 247 & 60002 & Rhodococcus rubropertinctus ${ }^{h}$ & E3602 & (3) & Soil \\
\hline 248 & 60003 & Rhodococcus rubropertinctus ${ }^{h}$ & ATCC 25593 & (8) & Soil \\
\hline 249 & 60017 & Rhodococcus rubropertinctus ${ }^{h}$ & ATCC 19067 & (8) & $?$ \\
\hline 250 & 65001 & Rhodococcus roseus ${ }^{i}$ & ATCC 271 & (8) & $\dot{?}$ \\
\hline 251 & 65002 & Rhodococcus roseus ${ }^{i}$ & ATCC 4004 (type) & (8) & $\dot{q}$ \\
\hline 252 & E7569 & Nocardia rubra & N25 & (14) & $?$ \\
\hline 253 & 23023 & Nocardia coeliaca & M-191 & (15) & $\dot{?}$ \\
\hline 254 & E7565 & Nocardia rubra & N19 & (14) & $?$ \\
\hline 255 & E7571 & Nocardia rubra & N27 & (14) & $\dot{?}$ \\
\hline 256 & 40001 & Rhodococcus rhodochrous* & & (3) & $?$ \\
\hline 257 & 50006 & Rhodococcus bronchialis & E3413 & (3) & Sputa, humans \\
\hline 258 & 50019 & Rhodococcus bronchialis & E3926 & (3) & Sputa, humans \\
\hline 259 & 50003 & Rhodococcus bronchialis & ATCC 25592 (type) & (8) & Sputa, humans \\
\hline 260 & 50011 & Rhodococcus bronchialis ${ }^{j}$ & E3885 & (3) & Sputa, humans \\
\hline 261 & 50017 & Rhodococcus bronchialis' & E3924 & (3) & Sputa, humans \\
\hline 262 & 23088 & Nocardia asteroides ${ }^{k}$ & M206 & (15) & $?$ \\
\hline 263 & 23044 & Nocardia asteroides ${ }^{k}$ & M124 & (15) & $?$ \\
\hline 264 & 23095 & Nocardia asteroides ${ }^{k}$ & $\mathrm{R} 433(1)$ & (10) & $?$ \\
\hline 265 & 23009 & Nocardia asteroides ${ }^{k}$ & M124 & (15) & ? \\
\hline 266 & 23032 & Nocardia asteroides ${ }^{k}$ & M10 & (15) & $?$ \\
\hline 267 & 23038 & Nocardia asteroides ${ }^{k}$ & M93 & (15) & $\dot{?}$ \\
\hline 268 & 23097 & Nocardia asteroides ${ }^{k}$ & R553 & (10) & $\dot{?}$ \\
\hline 269 & 23103 & Nocardia asteroides ${ }^{k}$ & R9970 & (10) & $\dot{?}$ \\
\hline 270 & 23045 & Nocardia asteroides ${ }^{k}$ & M128 & (15) & $\dot{?}$ \\
\hline 271 & 23057 & Nocardia asteroides ${ }^{k}$ & M156 & (15) & ? \\
\hline 272 & 23006 & Nocardia asteroides ${ }^{k}$ & M93 & (15) & ? \\
\hline 273 & 23007 & Nocardia asteroides ${ }^{k}$ & M94 & (15) & $\dot{?}$ \\
\hline 274 & 23039 & Nocardia asteroides ${ }^{k}$ & M94 & (15) & $?$ \\
\hline 275 & 23049 & Nocardia asteroides ${ }^{k}$ & M132 & (15) & $?$ \\
\hline 276 & 23054 & Nocardia asteroides ${ }^{k}$ & M145 & (15) & $?$ \\
\hline 277 & 23056 & Nocardia asteroides ${ }^{k}$ & M155 & (15) & ? \\
\hline 278 & 23046 & Nocardia asteroides ${ }^{k}$ & M129 & (15) & $?$ \\
\hline 279 & 23012 & Nocardia asteroides ${ }^{k}$ & M145 & (15) & ? \\
\hline 280 & 23105 & Nocardia brasiliensis & R432 & (10) & ? \\
\hline 281 & 23106 & Nocardia brasiliensis & R887 & (10) & $\dot{?}$ \\
\hline 282 & 23108 & Nocardia brasiliensis & R1117A & (10) & $?$ \\
\hline 283 & 23083 & Nocardia brasiliensis & M199 & (15) & $\dot{?}$ \\
\hline 284 & 23112 & Nocardia caviae & R547 & (10) & $\dot{?}$ \\
\hline 285 & 23113 & Nocardia caviae & R617 & (10) & $\dot{?}$ \\
\hline 286 & 23114 & Nocardia caviae & R1291 & (10) & ? \\
\hline 287 & 23033 & Nocardia caviae & M54 & (15) & $?$ \\
\hline 288 & 23035 & Nocardia caviae & M73 & (15) & ? \\
\hline
\end{tabular}


TABLE 1 (continued)

\begin{tabular}{|c|c|c|c|c|c|}
\hline \multirow{2}{*}{$\begin{array}{c}\text { Serial } \\
\text { no. }\end{array}$} & \multirow{2}{*}{$\begin{array}{l}\text { Labora- } \\
\text { tory no. }\end{array}$} & \multicolumn{2}{|c|}{ Received as: } & \multirow{2}{*}{ Source ${ }^{\prime}$} & \multirow{2}{*}{ Habitat } \\
\hline & & Name & Strain & & \\
\hline 289 & 23078 & Nocardia caviae & M185 & (15) & $?$ \\
\hline 290 & 23111 & Nocardia caviae & R416 & (10) & $?$ \\
\hline 291 & 23109 & Nocardia brasiliensis & $\mathrm{R} 1188$ & (10) & $?$ \\
\hline 292 & 23005 & Nocardia caviae & M73 & (15) & $?$ \\
\hline 293 & 23086 & Nocardia brasiliensis & M204 & (15) & $?$ \\
\hline 294 & 23067 & Nocardia farcinica ${ }^{k}$ & M164 & (15) & $?$ \\
\hline 295 & 23070 & Nocardia farcinica ${ }^{k}$ & M169 & (15) & $?$ \\
\hline 296 & 23051 & Nocardia farcinica ${ }^{k}$ & M138 & (15) & $?$ \\
\hline 297 & 23060 & Nocardia farcinica ${ }^{k}$ & M127 & (15) & $?$ \\
\hline 298 & 23082 & Nocardia farcinica ${ }^{k}$ & M198 & (15) & ? \\
\hline 299 & 23098 & Nocardia farcinica ${ }^{k}$ & R784 & (10) & $?$ \\
\hline 300 & 23048 & Nocardia farcinica ${ }^{k}$ & M131 & (15) & $?$ \\
\hline 301 & 23058 & Nocardia farcinica ${ }^{k}$ & M158 & (15) & $?$ \\
\hline 302 & 23065 & Nocardia farcinica ${ }^{k}$ & M162 & (15) & $?$ \\
\hline 303 & 23075 & Nocardia farcinica ${ }^{k}$ & M179 & (15) & $?$ \\
\hline 304 & 23081 & Nocardia farcinica ${ }^{k}$ & M197 & (15) & $?$ \\
\hline 305 & 23084 & Nocardia farcinica ${ }^{k}$ & M201 & (15) & $?$ \\
\hline 306 & 23101 & Nocardia farcinica & W3409B & (10) & ? \\
\hline 307 & 23041 & Nocardia farcinica ${ }^{k}$ & $\mathrm{M} 112$ & (15) & ? \\
\hline 308 & 23004 & Nocardia farcinica ${ }^{k}$ & M72 & (15) & $?$ \\
\hline 309 & 23034 & Nocardia farcinica ${ }^{k}$ & M72 & (15) & $?$ \\
\hline 310 & 23011 & Nocardia farcinica ${ }^{k}$ & M128 & (15) & $?$ \\
\hline 311 & 23013 & Nocardia farcinica ${ }^{k}$ & M146 & (15) & ? \\
\hline 312 & 23040 & Nocardia farcinica ${ }^{k}$ & M111 & (15) & $?$ \\
\hline 313 & 23102 & Nocardia farcinica ${ }^{k}$ & R3318 (type) & (10) & $?$ \\
\hline 314 & 23053 & Nocardia farcinica ${ }^{k}$ & M144 & (15) & $?$ \\
\hline 315 & E7551 & Nocardia farcinica* & VAC462 & (16) & $?$ \\
\hline 316 & 23001 & Nocardia farcinica ${ }^{k}$ & M75 & (15) & $?$ \\
\hline 317 & 23027 & Nocardia farcinica ${ }^{k}$ & C743 & (15) & $?$ \\
\hline 318 & 23087 & Nocardia farcinica ${ }^{k}$ & M205 & (15) & $?$ \\
\hline 319 & 23055 & Nocardia farcinica ${ }^{k}$ & M146 & (15) & $?$ \\
\hline 320 & 23018 & Nocardia farcinica ${ }^{k}$ & $\mathrm{C} 75$ & (15) & $?$ \\
\hline 321 & 23059 & Nocardia farcinica ${ }^{k}$ & M86 & (15) & $?$ \\
\hline 322 & 23062 & Nocardia farcinica ${ }^{k}$ & M158A & (15) & ? \\
\hline 323 & 23092 & Nocardia farcinica ${ }^{k}$ & M189 & (15) & ? \\
\hline 324 & 23010 & Nocardia farcinica ${ }^{k}$ & M126 & (15) & ? \\
\hline 325 & 23073 & Nocardia farcinica ${ }^{k}$ & M175 & (15) & $?$ \\
\hline 326 & E7550 & Nocardia farcinica & VAC333 & (16) & $?$ \\
\hline 327 & 23069 & Nocardia farcinica ${ }^{k}$ & M167 & (15) & $?$ \\
\hline 328 & 23074 & Nocardia farcinica ${ }^{k}$ & M177 & (15) & ? \\
\hline 329 & 23093 & Nocardia farcinica ${ }^{k}$ & M200 & (15) & $?$ \\
\hline 330 & E7547 & Nocardia farcinica* & VAC300 & (16) & $?$ \\
\hline 331 & E7548 & Nocardia farcinica* & VAC324 & $(16)$ & ? \\
\hline 332 & E7549 & Nocardia farcinica* & VAC330 & $(16)$ & ? \\
\hline 333 & 23077 & Nocardia farcinica ${ }^{k}$ & M181 & (15) & $?$ \\
\hline 334 & E7815 & Nocardia farcinica* & 3318/AL & (11) & $?$ \\
\hline 335 & 23104 & Nocardia brasiliensis & R405 & $(10)$ & $?$ \\
\hline 336 & E7723 & Corynebacterium equi & $\mathrm{KO}-85$ & (17) & $?$ \\
\hline 337 & E3477 & Rhodococcus aurantiacus' & & (3) & Sputa, humans \\
\hline 338 & 80001 & Rhodococcus aurantiacus ${ }^{l}$ & ATCC 25938 (type) & (8) & Sputa, humans \\
\hline 339 & 80003 & Rhodococcus aurantiacus ${ }^{I}$ & E4409 & (3) & Sputa, humans \\
\hline 340 & E4134 & Rhodococcus aurantiacus ${ }^{l}$ & & (3) & Sputa, humans \\
\hline 341 & E4259 & Rhodococcus aurantiacus ${ }^{l}$ & & (3) & Sputa, humans \\
\hline 342 & E7552 & Rhodococcus aurantiacus ${ }^{l}$ & 25938 & (16) & Sputa, humans \\
\hline 343 & E6618 & Jensenia canicruria & KCCA-0132 & (18) & $?$ \\
\hline 344 & 40014A & Jensenia canicruria & KCCA-0132 & (18) & $?$ \\
\hline 345 & 40014 & Jensenia canicruria & KCCA-0132 (type) & (18) & $?$ \\
\hline 346 & 23066 & ? & M163 & (15) & ? \\
\hline 347 & 23085 & Nocardia tenuis-convoluta & M203 & (15) & $?$ \\
\hline 348 & $\mathrm{E} 6222$ & Mycobacterium agri & & (3) & Soil \\
\hline
\end{tabular}


TABLE 1 (continued)

\begin{tabular}{|c|c|c|c|c|c|}
\hline \multirow{2}{*}{$\begin{array}{l}\text { Serial } \\
\text { no. }\end{array}$} & \multirow{2}{*}{$\begin{array}{l}\text { Labora- } \\
\text { tory no. }\end{array}$} & \multicolumn{2}{|c|}{ Received as: } & \multirow{2}{*}{ Source ${ }^{a}$} & \multirow{2}{*}{ Habitat } \\
\hline & & Name & Strain & & \\
\hline 349 & 90001 & Mycobacterium agri & E6203 & (3) & Soil \\
\hline 350 & 90012 & Mycobacterium agri & ATCC 27406 (type) & (8) & Soil \\
\hline 351 & 90018 & Mycobacterium agri & ATCC 27407 & (8) & Soil \\
\hline 352 & 17002 & Mycobacterium smegmatis & SN2 & (12) & $?$ \\
\hline 353 & 17005 & Mycobacterium smegmatis & SN5 & (12) & $?$ \\
\hline 354 & 17023 & Mycobacterium smegmatis* & Jucho & (19) & Hens \\
\hline 355 & 17027 & Mycobacterium smegmatis & $\begin{array}{l}\text { ATCC } 14468 \text { (neo- } \\
\text { type) }\end{array}$ & (8) & $?$ \\
\hline 356 & 43001 & $\begin{array}{l}\text { Mycobacterium smegmatis (bu- } \\
\text { tyricum) }\end{array}$ & ATCC 19979 & (8) & $?$ \\
\hline 357 & E7817 & $?$ & IP740 & (11) & $?$ \\
\hline 358 & E7819 & $?$ & N10955 & (11) & $?$ \\
\hline 359 & 23031 & Proactinomyces restrictus & M9 & (15) & $?$ \\
\hline 360 & E7816 & $?$ & IP736 & (11) & $?$ \\
\hline 361 & E7729 & Corynebacterium ulcerans & 9304 & (17) & $?$ \\
\hline 362 & 23030 & Nocardia polychromogenes & M6 & (15) & $?$ \\
\hline 363 & E7955 & $?$ & B-11-25-LD & (7) & Swine, thelitis lesions \\
\hline 364 & E7956 & $?$ & B-11-25-OD & (7) & Swine, thelitis lesions \\
\hline 365 & E7724 & Corynebacterium hoffmanii & 39 & (17) & ? \\
\hline 366 & 21007 & Mycobacterium vaccae & ATCC 15483 (type) & $(8)$ & Cow's milk \\
\hline 367 & 21011 & Mycobacterium vaccae & ATCC 23014 & (8) & ? \\
\hline 368 & 21012 & Mycobacterium vaccae & ATCC 23027 & (8) & $?$ \\
\hline 369 & E7728 & Corynebacterium diphtheriae & PW8 & (17) & $?$ \\
\hline
\end{tabular}

${ }^{a}$ (1) H. H. Kleeberg, Tuberculosis Research Institute, Pretoria, South Africa; (2) S. Ata, Nagoya University, Nagoya, Japan; (3) M. Tsukamura, National Chubu Hospital, Obu, Aichi, Japan; (4) K. Shoji, Osaka University, Osaka, Japan; (5) K. Konno, Tohoku University, Sendai, Japan; (6) J. Marks, Tuberculosis Reference Laboratory, University Hospital of Wales, Cardiff, Wales; (7) K. Shimizu, Obihiro Veterinary College, Hokkaido, Japan; (8) ATCC, American Type Culture Collection, Rockville, Md., U.S.A.; (9) K. Takeya, Kyushu University, Fukuoka, Japan; (10) R. E. Gordon, Rutgers University, New Brunswick, N.J., U.S.A.; (11) M. P. Lechevalier, Rutgers University, New Brunswick, N.J., U.S.A.; (12) R. Bönicke, Forschungsinstitut Borstel, Borstel, West Germany; (13) H. Saito, Hiroshima University, Hiroshima, Japan; (14) M. Riddel, University of Göteborg, Göteborg, Sweden; (15) I. Uesaka, Kyoto University, Kyoto, Japan (originally received from N. M. McClung, University of South Florida, Tampa, Fl., U.S.A.); (16) S. G. Bradley, Virginia Commonwealth University, Richmond, Va., U.S.A.; (17) M. Yoneda, Osaka University, Osaka, Japan; (18) A. Seino, Kaken Chemical Co., Tokyo, Japan; (19) T. Hasegawa, Tokyo University, Tokyo, Japan.

${ }^{b}$ Strains marked with an asterisk were identified in this laboratory.

' Mycobacterium chelonei subsp. abscessus should be named $M$. abscessus (16).

${ }^{d}$ Mycobacterium chelonei subsp. chelonei should be named $M$. chelonei (16) (M. borstelense is a synonym of this organism).

'Formerly Gordona lentifragmenta (synonym: Nocardia rubra) (26); see reference 20.

${ }^{f}$ Formerly Gordona rhodochroa (19); see reference 20.

"Formerly Gordona terrae (18); see reference 20.

${ }^{\text {h}}$ Formerly Gordona rubropertincta (synonym Gordona rubra) $(18,19)$; refer to (20).

' Formerly Gordona rosea $(19,20)$.

${ }^{j}$ Formerly Gordona bronchialis $(18,20)$.

${ }^{k}$ Identified in this laboratory (15); for the names as received, see reference 15.

' Formerly Gordona aurantiaca $(20,25)$.

laboratory, omitting, as far as possible, linked characters, which are considered to give unreliable results (23). The characters were the same as those used previously for a numerical classification of slowly growing mycobacteria (22). Taken as a whole, the 88 characters were effective for differentiating the strains.

Numerical analysis. The matching coefficient ( $M$ value) between any two strains was calculated by the following equation: $M$ value (percent) $=\left(n_{s} \times 100 \%\right) /$ $\left(n_{s}+n_{d}\right)$, where $n_{s}$ is the number of characters that show similar code symbols $(++$ or --$)$ and $n_{d}$ is the number of characters which show different code symbols $(+-)$. Clustering was done by the single-linkage method (12).

The computer used was the IBM S-370, model 135, which is located in the Nagoya Factory of the Sumitomo Machinery Co., Obu, Aichi, Japan. The program is written in English (CŌBOLL), and the program number is G90002. The data for the original "strain $\times$ characters" matrix have been deposited with the World Data Center for Microorganisms, Brisbane, Australia. 
In the numerical classification, two or three replicates of a few strains were included in the study to confirm the reliability of the results.

\section{RESULTS}

The results of the study are presented as dendrograms (Fig. 1 to 7 ). Of the 369 strains studied, 346 strains formed a large cluster at a level of $84 \%$; this cluster was subdivided into four smaller clusters at a level of $85 \%$.

The first cluster (A) corresponded to the group of organisms referred to as slowly growing mycobacteria; the second (B), to intermediates between slowly and rapidly growing mycobacteria; the third $(\mathrm{C})$, to rapidly growing mycobacteria; and the fourth (D), to rhodococci and nocardiae. The remaining 23 strains, including those of Mycobacterium agri, $M$. smegmatis, and $M$. vaccae, were located outside of these four clusters. Each of these clusters is discussed below.

Cluster A (strains 1-167). In cluster A (Fig.
1, 2, and 3; slowly growing mycobacteria), the tuberculosis (TB) complex, consisting of $M y c o$ bacterium tuberculosis, $M$. bovis, $M$. africanum, and $M$. microti, formed a compact subcluster at a level of $97 \%$. Within this subcluster, strains of $M$. tuberculosis could be distinguished from strains of the other three species, but these could not be distinguished fróm each other (Fig. 1).

In cluster $\mathrm{A}$, the following 11 subclusters were evident: 1 , TB complex; $2, M$. avium-M. intracellulare-M. scrofulaceum complex; $3, M$. gordonae; 4, M. xenopi; 5, M. nonchromogenicum; 6, M. gastri; 7, M. kansasii; 8, M. szulgai; $9, M$. shimoide $i$; $10, M$. marinum; and 11 , strains 165 and 166. These subclusters were formed at levels between 92 and $98 \%$. A close relationship between subclusters $2(M$. avium $-M$. intracellulare-M. scrofulaceum complex) and 3 (M. gordonae) was observed. These were combined into a single cluster at a level of $93 \%$. Subclusters 2 to 11 , inclusive, formed a single cluster at a level of $89 \%$ and were linked together with subcluster 1 (TB complex) at a level of $85 \%$.

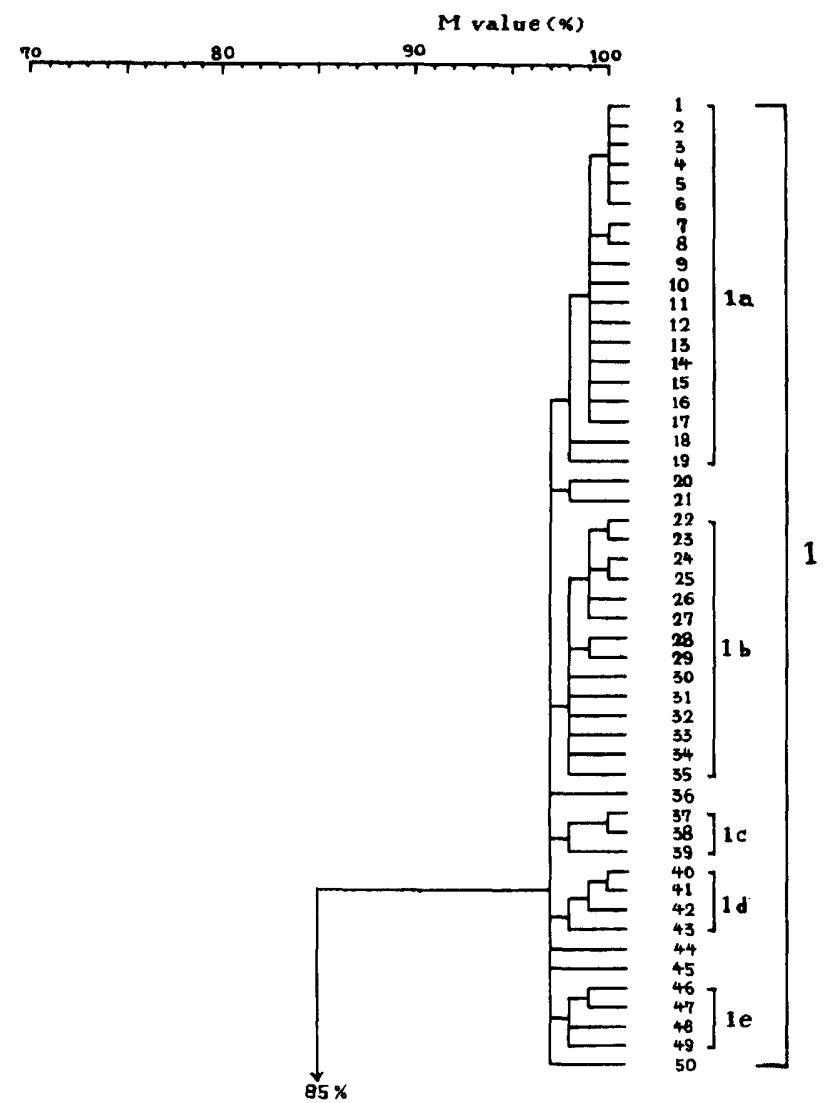

Fig. 1. Dendrogram (part 1). Subcluster 1, TB complex; 1a, M. tuberculosis; 1b, M. africanum and M. bovis; 1c, $M$. bovis; 1d, M. bovis; le, M. microti. 


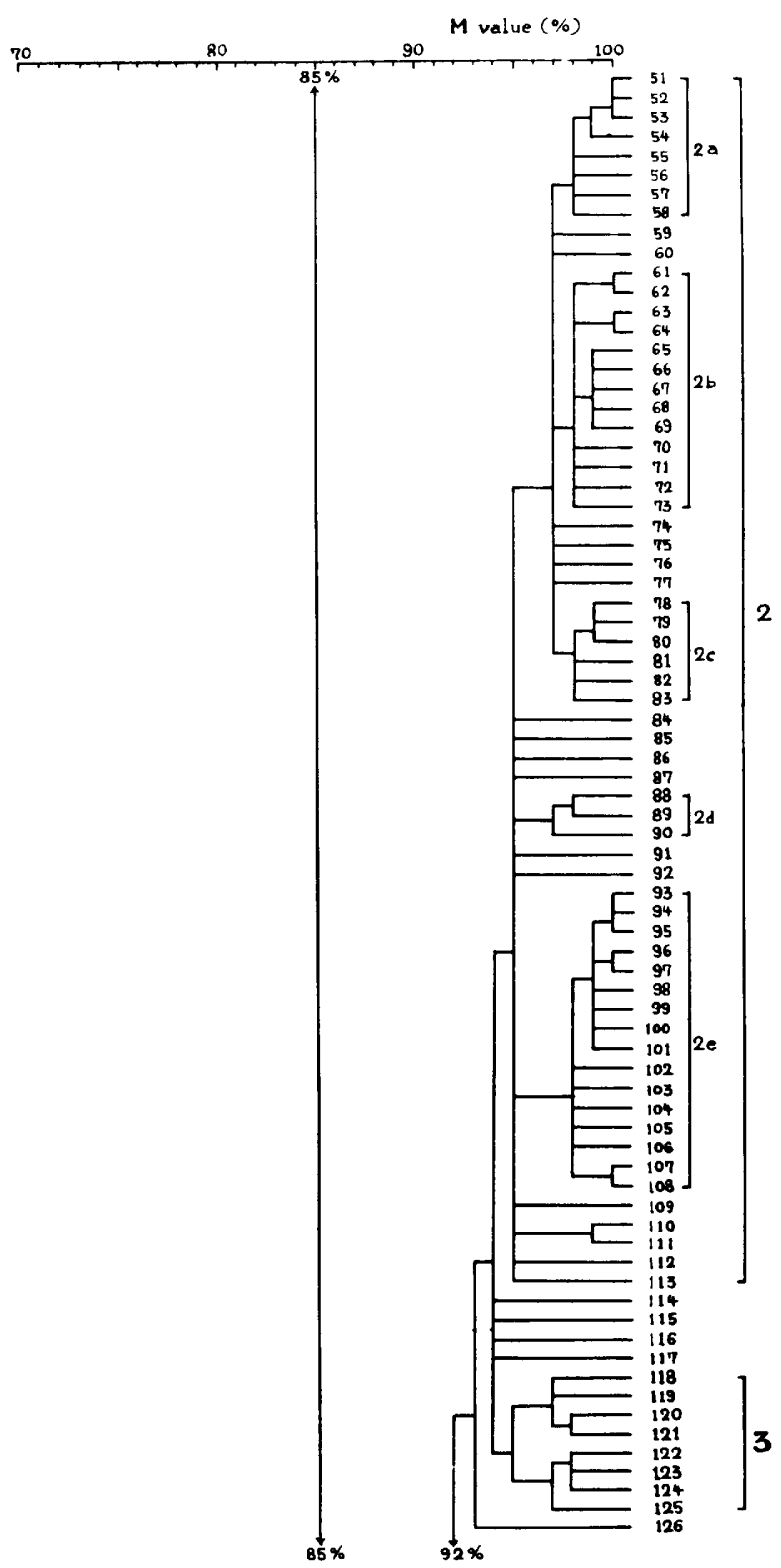

Fig. 2. Dendrogram (part 2). Subcluster 2, M. avium-M. intracellulare-M. scroflaceum complex; 2a, Marks's strains of $M$. intracellulare; $2 \mathrm{~b}, M$. intracellulare; $2 \mathrm{c}, M$. avium; $2 \mathrm{~d}, M$. intracellulare; $2 \mathrm{e}, \boldsymbol{M}$. scrofulaceum; 3, M. gordonae.

Cluster B (strains 168-175). Cluster B (Fig. 3) was composed of strains of only two mycobacterial species, $M$. flavescens (subcluster 12) and $M$. thermoresistibile (subcluster 12). These two subclusters consisted of strains intermediate between the slowly growing and the rapidly growing mycobacteria.

Cluster C (strains 176-225). Cluster C (Fig. 4) was composed of the following subclusters
(14-22, inclusive) of rapidly growing mycobacteria: $14, M$. fortuitum; $15, M$. aurum; $16, M$. abscessus (M. chelonei subsp. abscessus); 17, $M$. chelonei (M. chelonei subsp. chelonei); 18, $M$. phlei; 19, M. parafortuitum; 20, M. chubuense; $21, M$. aichiense; and 22, M. chitae. (M. rhodesiae was not shown as a subcluster, because only a single strain was tested in this study.)

Subclusters 14 to 22 , inclusive, were distinctly 


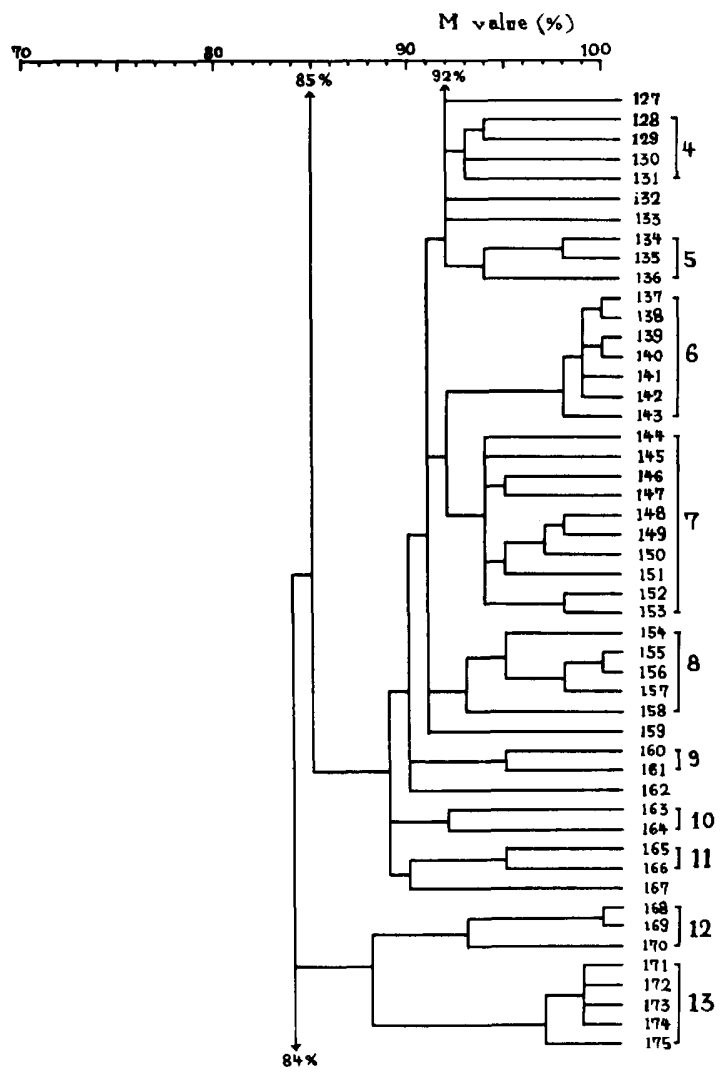

Fig. 3. Dendrogram (part 3). Subcluster 4, M. xenopi; $5, M$. nonchromogenicum; $6, M$. gastri; $7, M$. kansasii; 8, M. szulgai; 9 , M. shimoidei; 10, M. marinum; 11 , strains 165 and $166 ; 12, M$. flavescens; $13, M$. thermoresistible.

differentiated from each other at a level of $90 \%$ and were combined into a single cluster, $\mathrm{C}$, at a level of $85 \%$.

Cluster D (strains 226-345). Cluster D (Fig. 5 and 6) were composed of subclusters (23-33, inclusive) of strains of the following species of the genera Rhodococcus and Nocardia: 23, Rhodococcus lentifragmentus (formerly Gordona lentifragmenta [26]; according to Goodfellow and Alderson [3], R. ruber; synonym: Nocardia rubra); 24, $R$. rhodochrous (synonym: Gordona rhodochroa); 25a, R. terrae (synonym: Gordona terrae); 25b, $R$. rubropertinctus (synonyms: Gordona rubropertincta and G. rubra); 25c, $R$. roseus (synonym: Gordona rosea); 26, $R$. bronchialis (synonym: Gordona bronchialis); 27, Nocardia asteroides B (23); $28, N$. asteroides A (23); 29, N. brasiliensis; 30, N. caviae; $31, N$. farcinica; 32, $R$. aurantiacus (synonym: Gordona aurantiaca); and 33, Jensenia canicruria (three replicates from a single strain).

Cluster D contained two large subclusters, D1 (from 23-27, Fig. 5) and D2 (from 28-31, Fig. 6), the former consisting of $R$. lentifragmentus to
$N$. asteroides B and the latter consisting of $N$. asteroides A to $N$. farcinica. Subclusters D1 and D2 corresponded to rhodococci and nocardiae, respectively, although some strains of $N$. asteroides were located in the former. Strains of $R$. aurantiacus and $J$. canicruria were located outside of these two large subclusters. The single strain of Corynebacterium equi was incorporated into cluster $D$.

Distinguishing characters. Characters useful for differentiating clusters A, B, C, and D from each other are given in Table 2. Distinctive characters of the species in each cluster are presented in Tables 3 to 5 .

\section{DISCUSSION}

In the present study, four major clusters of the 369 strains studied were observed (Fig. 7). These clusters were composed of: (A) slowly growing mycobacteria; (B) intermediate mycobacteria; (C) rapidly growing mycobacteria; and (D) rhodococci and nocardiae. Previously, Tsukamura $(17,21)$ suggested that slowly growing 
Fig. 4. Dendrogram (part 4). Subcluster 14, $M$. fortuitum; $15, M$. aurum; $16, M$. abscessus (M. chelonei subsp. abscessus); 17, M. chelonei (M. chelonei susp. chelonei); $18, M$. phlei; 19 , $M$. parafortuitum; 20, M. chubuense; $21, M$. aichiense; 22, M. chitae.

Fig. 5. Dendrogram (part 5). Subcluster 23, $R$. lentifragmentus; 24, $R$. rhodochrous; 25a, $R$. terrae; 25b, $R$. rubropertinctus; $25 \mathrm{c}, R$. roseus; $26, R$. bronchialis; $27, N$. asteroides B.
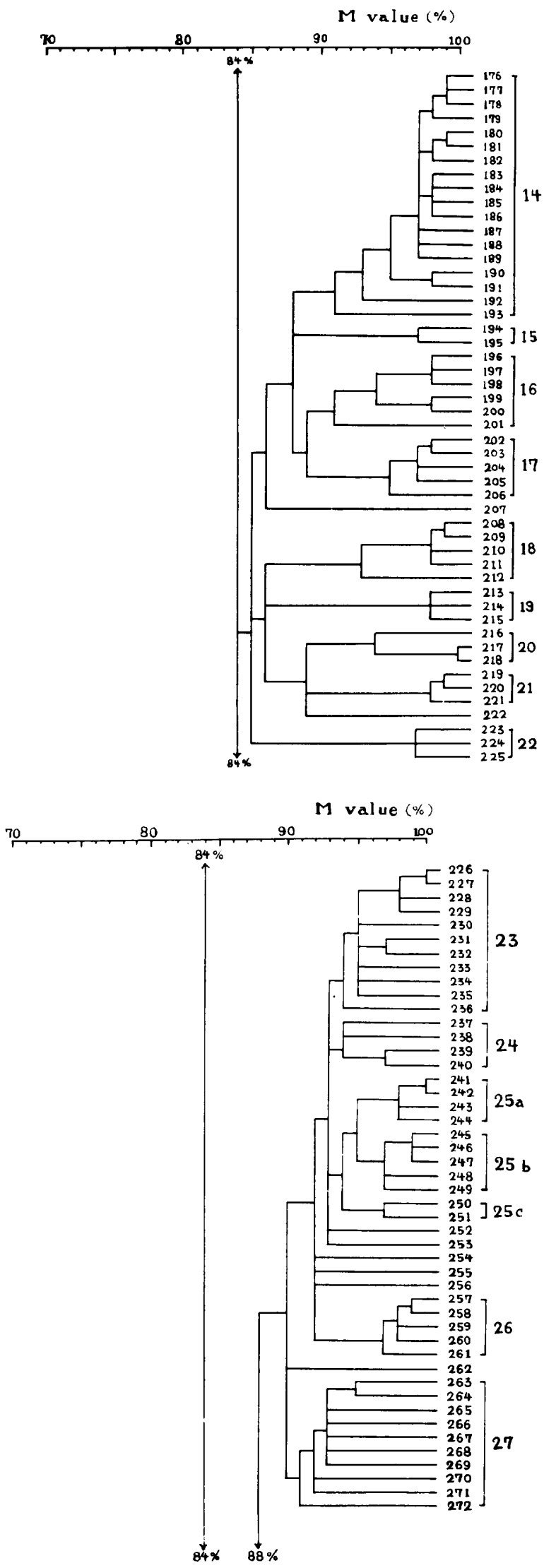


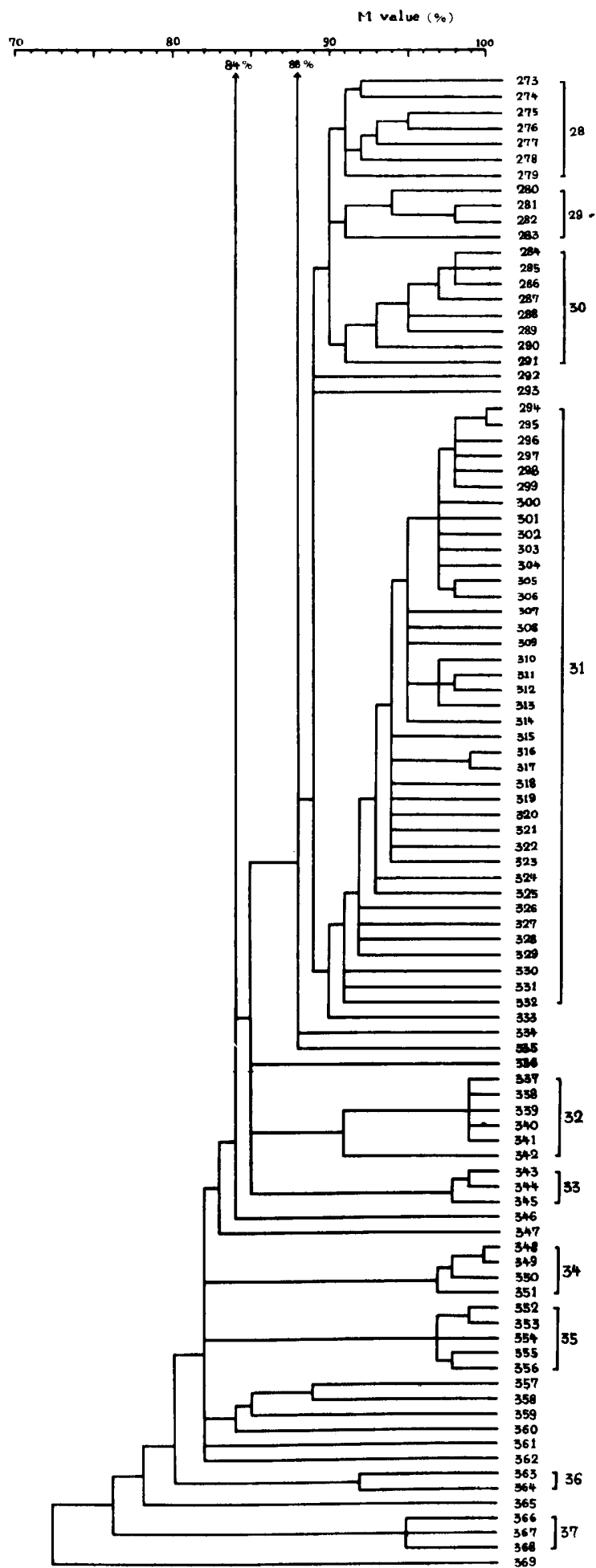

Fig. 6. Dendrogram (part 6). Subcluster 28, N. asteroides A; 29, N. brasiliensis; 30, N. caviae; $31, N$. farcinica; $32, R$. aurantiacus; 33 , J. canicruria; $34, M$. agri; $35, M$. smegmatis; 36 , mycobacterial strains 363 and $364 ; 37, M$. vaccae. 


\begin{tabular}{|c|c|c|c|c|c|c|c|c|c|c|c|c|c|c|c|c|c|c|}
\hline \multirow[b]{2}{*}{ 迹 } & \multirow[b]{2}{*}{ 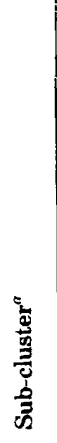 } & \multirow[b]{2}{*}{ 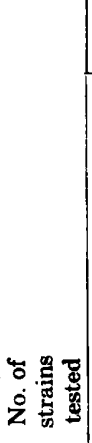 } & \multicolumn{16}{|c|}{ No. of strains showing positive reaction } \\
\hline & & & 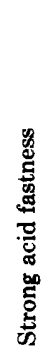 & 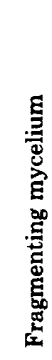 & 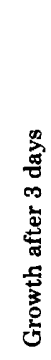 & 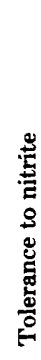 & 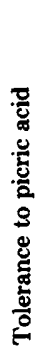 & 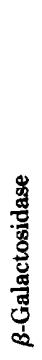 & 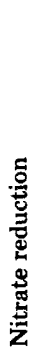 & 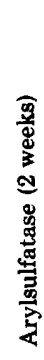 & 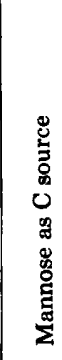 & 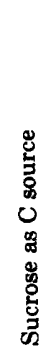 & 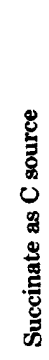 & 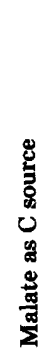 & 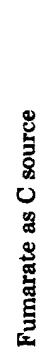 & 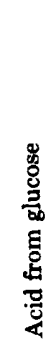 & 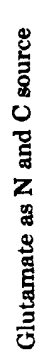 & 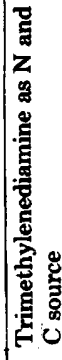 \\
\hline \multirow[t]{11}{*}{$\mathbf{A}$} & 1 & 50 & 50 & 0 & 0 & 0 & 0 & 0 & 21 & 22 & 0 & 0 & 0 & 0 & 0 & 0 & 0 & 0 \\
\hline & 2 & 63 & 63 & 0 & 0 & 0 & 0 & 0 & 0 & 52 & 0 & 0 & 0 & 0 & 0 & 0 & 3 & 0 \\
\hline & 3 & 8 & 8 & $\mathbf{0}$ & $\mathbf{0}$ & $\mathbf{0}$ & 0 & 0 & $\mathbf{0}$ & 8 & $\mathbf{0}$ & $\mathbf{0}$ & $\mathbf{0}$ & 0 & $\mathbf{0}$ & $\mathbf{0}$ & 1 & 0 \\
\hline & 4 & 4 & 4 & 0 & 0 & 0 & 0 & 0 & 0 & 4 & 0 & 0 & 0 & 0 & 0 & 0 & 0 & 0 \\
\hline & 5 & 3 & 3 & 0 & 0 & 1 & 0 & 3 & 0 & 3 & 0 & 0 & 0 & 0 & 0 & 0 & 0 & 0 \\
\hline & 6 & 7 & 7 & 0 & 0 & 0 & 0 & 0 & 7 & 7 & 0 & 0 & 0 & 0 & 0 & 0 & 0 & 0 \\
\hline & 7 & 10 & 10 & 0 & 0 & 0 & 0 & 0 & 10 & 10 & 0 & 0 & 0 & 0 & 0 & 0 & 5 & 0 \\
\hline & 8 & 5 & 5 & 0 & 0 & 0 & 0 & 0 & 5 & 4 & 0 & 0 & 0 & 0 & 0 & 0 & 0 & 0 \\
\hline & 9 & 2 & 2 & 0 & 0 & 0 & 0 & 0 & 0 & 0 & 0 & 0 & 0 & 0 & 0 & 0 & 0 & 0 \\
\hline & 10 & 2 & 2 & 0 & 0 & 0 & 0 & 0 & 0 & 2 & 0 & 0 & 0 & 0 & 0 & 0 & 2 & 0 \\
\hline & 11 & 2 & 2 & 0 & 0 & 0 & 0 & 0 & 0 & 2 & 0 & 0 & 0 & 0 & 0 & 0 & 0 & 0 \\
\hline \multirow[t]{2}{*}{ B } & 12 & 3 & 3 & 0 & 3 & 3 & 3 & 0 & 3 & 3 & 3 & 0 & 2 & 0 & 0 & 2 & 3 & 0 \\
\hline & 13 & 5 & 5 & 0 & 5 & 5 & 5 & 0 & 5 & 0 & 0 & 0 & 2 & 4 & 5 & 0 & 5 & 0 \\
\hline \multirow[t]{9}{*}{$\mathrm{C}$} & 14 & 18 & 18 & 0 & 18 & 18 & 18 & 0 & 18 & 18 & 18 & 0 & 18 & 18 & 18 & 18 & 18 & 18 \\
\hline & 15 & 2 & 2 & 0 & 2 & 2 & 2 & 0 & 2 & 2 & 2 & 0 & 2 & 2 & 2 & 2 & 2 & 2 \\
\hline & 16 & 6 & 6 & 0 & 6 & 6 & 6 & 6 & 0 & 6 & 6 & 0 & 6 & 6 & 4 & 4 & 6 & 6 \\
\hline & 17 & 5 & 5 & 0 & 5 & 5 & 0 & 5 & 0 & 5 & 5 & 0 & 1 & 0 & 1 & 5 & 5 & 0 \\
\hline & 18 & 5 & 5 & 0 & 5 & 5 & 5 & 5 & 5 & 5 & 5 & 1 & 5 & 5 & 5 & 5 & 5 & 5 \\
\hline & 19 & 3 & 3 & 0 & 3 & 3 & 3 & 0 & 2 & 3 & 3 & 3 & 3 & 3 & 3 & 3 & 3 & 3 \\
\hline & 20 & 3 & 3 & 0 & 3 & 3 & 3 & 0 & 3 & 3 & 3 & 3 & 3 & 3 & 3 & 3 & 3 & 3 \\
\hline & 21 & 3 & 3 & 0 & 3 & 3 & 3 & 0 & 0 & 3 & 3 & 0 & 3 & 3 & 3 & 3 & 3 & 0 \\
\hline & 22 & 3 & 3 & 0 & 3 & 3 & 3 & 0 & 3 & 3 & 3 & 0 & 0 & 0 & 0 & 3 & 3 & 0 \\
\hline \multirow[t]{15}{*}{$\mathbf{D}$} & 23 & 11 & 0 & 9 & 11 & 11 & 11 & 0 & 10 & 0 & 11 & 5 & 11 & 11 & 11 & 11 & 11 & 0 \\
\hline & 24 & 4 & 0 & 1 & 4 & 4 & 4 & 0 & 4 & 0 & 4 & 4 & 3 & 4 & 4 & 4 & 4 & 0 \\
\hline & 25 & 11 & 0 & 0 & 11 & 11 & 11 & 0 & 11 & 0 & 11 & 11 & 11 & 11 & 11 & 11 & 11 & 0 \\
\hline & 26 & 5 & 0 & 0 & 5 & 5 & 5 & 0 & 5 & 0 & 5 & 5 & 5 & 5 & 5 & 5 & 5 & 0 \\
\hline & 27 & 10 & 0 & 10 & 10 & 10 & 10 & 10 & 7 & 4 & 1 & 0 & 8 & 7 & 9 & 6 & 10 & 0 \\
\hline & 28 & 7 & 0 & 7 & 7 & 7 & 7 & 7 & 2 & 0 & 2 & 0 & 7 & 7 & 6 & 2 & 7 & 0 \\
\hline & 29 & 4 & 0 & 4 & 4 & 4 & 4 & 4 & 0 & 0 & 3 & 0 & 4 & 4 & 4 & 4 & 4 & 0 \\
\hline & 30 & 8 & 0 & 8 & 8 & 8 & 8 & 8 & 0 & 0 & 8 & 1 & 8 & 8 & 8 & 8 & 8 & 0 \\
\hline & 31 & 39 & 0 & 39 & 39 & 25 & 38 & 39 & 1 & 0 & 38 & 12 & 39 & 37 & 38 & 39 & 39 & 0 \\
\hline & 32 & 6 & 0 & 0 & 6 & 6 & 6 & 6 & 1 & 0 & 6 & 6 & 6 & 6 & 6 & 6 & 6 & 0 \\
\hline & 33 & 3 & 0 & 0 & 3 & 3 & 3 & 0 & 0 & 0 & 3 & 3 & 3 & 3 & 3 & 3 & 1 & 0 \\
\hline & 34 & 4 & 4 & 0 & 4 & 4 & 4 & 4 & 4 & 4 & 4 & 0 & 4 & 4 & 4 & 4 & 4 & 0 \\
\hline & 35 & 5 & 5 & 0 & 5 & 5 & 5 & 5 & 5 & 5 & 5 & 5 & 5 & 5 & 5 & 5 & 5 & 5 \\
\hline & 36 & 2 & 2 & 0 & 2 & 2 & 2 & 0 & 0 & 0 & 2 & 2 & 2 & 2 & 2 & 2. & 2 & 2 \\
\hline & 37 & 3 & 3 & 0 & 3 & 3 & 3 & 0 & 0 & 3 & 3 & 0 & 0 & 0 & 0 & 3. & 3 & 3 \\
\hline
\end{tabular}

${ }^{a}$ Subclusters: 1 , TB complex; $2, M$. avium-M. intracellulare- $M$. scrofulaceum complex; $3, M$. gordonae; $4, M$ xenopi; 5, M. nonchromogenicum; 6, M. gastri; $7, M$. kansasii; 8, M. szulgai; 9, M. shimoidei; 10, M. marinum; 11, mycobacterial strains 165 and $166 ; 12, M$. flavescens; $13, M$. thermoresisitibile; $14, M$. fortuitum; 15, M. aurum; $16, M$. abscessus; $17, M$. chelonei; $18, M$. phlei; 19, M. parafortuitum; $20, M$. chubuense; $21, M$. aichiense; $22, M$. chitae; $23, R$. lentifragmentus; $24, R$. rhodochrous; $25, R$. terrae- $R$. rubropertinctus- $R$. roseus; $26, R$. bronchialis; $27, N$. asteroides B; 28, N. asteroides A; $29, N$. brasiliensis; $30, N$. caviae; 31, N. farcinica; $32, R$. aurantiacus; $33, J$. canicruria; 34, M. agri; 35, $M$. smegmatis; 36 , mycobacterial strains 363 and $364 ; 37, M$. vaccae. 


\begin{tabular}{|c|c|c|c|c|c|c|c|c|c|}
\hline \multirow[b]{2}{*}{ Character } & \multicolumn{9}{|c|}{$\%$ of strains showing positive reaction } \\
\hline & 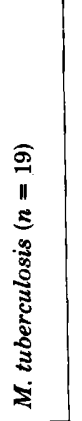 & 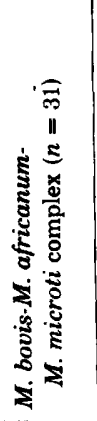 & 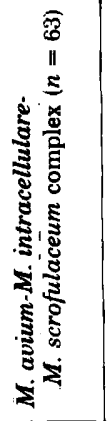 & 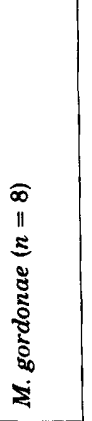 & 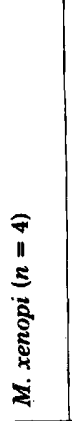 & 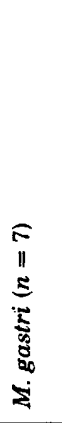 & 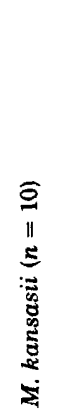 & 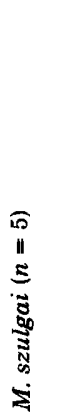 & 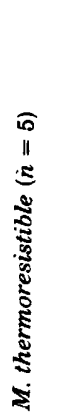 \\
\hline Rough colonies & 100 & 84 & 0 & 0 & 0 & 0 & 100 & 60 & 100 \\
\hline Photochromogenicity & 0 & 0 & 2 & 0 & 0 & 0 & 100 & 0 & 0 \\
\hline $\begin{array}{l}\text { Resistance to rifampin ( } 25 \\
\mu \mathrm{g} / \mathrm{ml})\end{array}$ & 0 & 0 & 100 & 50 & 75 & 14 & 0 & 20 & 100 \\
\hline $\begin{array}{l}\text { Resistance to } p \text {-aminosali- } \\
\text { cylic acid }(2 \mathrm{mg} / \mathrm{ml})\end{array}$ & 0 & 0 & 81 & 25 & 25 & 0 & $\mathbf{0}$ & 0 & 100 \\
\hline $\mathrm{NH}_{2} \mathrm{OH}-\mathrm{HCl}(0.125 \mathrm{mg} / \mathrm{ml})$ & 0 & 0 & 100 & 100 & 75 & 100 & 100 & 100 & 100 \\
\hline $\mathrm{NH}_{2} \mathrm{OH}-\mathrm{HCl}(0.25 \mathrm{mg} / \mathrm{ml})$ & 0 & 0 & 100 & 100 & 25 & 100 & 30 & 100 & 0 \\
\hline $\mathrm{NH}_{2} \mathrm{OH}-\mathrm{HCl}(0.5 \mathrm{mg} / \mathrm{ml})$ & 0 & 0 & 91 & 50 & $\mathbf{0}$ & 0 & $\mathbf{0}$ & 100 & $\mathbf{0}$ \\
\hline $\begin{array}{l}\text { Resistance to TCH }(10 \mu \mathrm{g} / \\
\text { ml) }\end{array}$ & 90 & 0 & 100 & 100 & 100 & 100 & 100 & 100 & 100 \\
\hline $\begin{array}{l}p \text {-Nitrobenzoic acid me- } \\
\text { dium }\end{array}$ & 0 & 0 & 100 & 100 & 100 & 100 & 100 & 100 & 100 \\
\hline Niacin & 100 & 58 & 0 & 0 & 0 & 0 & 0 & 0 & $\mathbf{0}$ \\
\hline Tween hydrolysis (14 days) & 0 & 0 & 2 & 100 & 100 & 100 & 90 & 40 & 100 \\
\hline$\alpha$-Esterase & 100 & 97 & 100 & 88 & 100 & 0 & 0 & 80 & 100 \\
\hline$\beta$-Esterase & 100 & 94 & 95 & 100 & 100 & $\mathbf{0}$ & 20 & 80 & 100 \\
\hline Acid phosphatase $(3 \mathrm{~h})$ & 0 & 0 & 79 & 0 & 0 & 100 & 90 & 100 & 100 \\
\hline Nitrate reduction $(24 \mathrm{~h})$ & 100 & 3 & 0 & 0 & 0 & 100 & 100 & 100 & 100 \\
\hline Arylsulfatase (2 weeks) & 0 & 74 & 81 & 100 & 100 & 100 & 100 & 100 & 0 \\
\hline Urease & 100 & 94 & 16 & 63 & $\mathbf{0}$ & 100 & 100 & 100 & 100 \\
\hline Nicotinamidase & 95 & 36 & 33 & 13 & 50 & 100 & 100 & 0 & 100 \\
\hline Pyrazinamidase & 89 & 19 & 33 & 13 & 50 & 0 & $\mathbf{0}$ & 0 & 100 \\
\hline Growth at $28^{\circ} \mathrm{C}$ & 5 & 26 & 100 & 100 & 0 & 100 & 100 & 100 & 100 \\
\hline Growth at $45^{\circ} \mathrm{C}$ & 0 & 0 & 65 & 0 & 100 & 0 & 0 & 0 & 100 \\
\hline Growth at $52^{\circ} \mathrm{C}$ & 0 & 0 & 0 & 0 & 0 & 0 & 0 & 0 & 100 \\
\hline $\begin{array}{l}\text { Glucose as } \mathrm{C} \text { source }\left(\mathrm{NH}_{3^{-}}\right. \\
\mathrm{N})\end{array}$ & 0 & 0 & 56 & 100 & $\mathbf{0}$ & 100 & 100 & 0 & 100 \\
\hline $\begin{array}{l}n \text {-Propanol as C source } \\
\left(\mathrm{NH}_{3}-\mathrm{N}\right)\end{array}$ & 0 & 0 & 65 & 100 & 0 & 100 & 30 & 0 & 100 \\
\hline $\begin{array}{l}\text { iso-Butanol as C source } \\
\left(\mathrm{NH}_{3}-\mathrm{N}\right)\end{array}$ & 0 & 0 & 41 & 100 & $\mathbf{0}$ & 86 & 40 & 0 & 100 \\
\hline $\begin{array}{l}n-\mathrm{Butanol} \text { as C source } \\
\left(\mathrm{NH}_{3}-\mathrm{N}\right)\end{array}$ & 0 & $\mathbf{0}$ & 44 & 88 & $\mathbf{0}$ & 86 & 40 & 0 & 100 \\
\hline $\begin{array}{l}\text { Acetate as } \mathrm{C} \text { source }\left(\mathrm{NH}_{3-}^{-}\right. \\
\text {N) }\end{array}$ & 0 & 0 & 97 & 100 & 75 & 100 & 50 & 100 & 100 \\
\hline $\begin{array}{l}\text { Pyruvate as } \mathrm{C} \text { source }\left(\mathrm{NH}_{3-}\right. \\
\text { N) }\end{array}$ & 0 & 0 & 94 & 100 & 75 & 100 & 100 & 100 & 100 \\
\hline Fumarate as $\mathrm{C}$ source & 0 & 0 & 0 & 0 & $\mathbf{0}$ & $\mathbf{0}$ & $\mathbf{0}$ & 0 & 100 \\
\hline $\begin{array}{l}\text { Glucose as C source (gluta- } \\
\text { mate-N) }\end{array}$ & 0 & 26 & 86 & 100 & 0 & 100 & 100 & 0 & 100 \\
\hline $\begin{array}{l}\text { Acetate as C source (gluta- } \\
\text { mate- } N \text { ) }\end{array}$ & 5 & 26 & 97 & 100 & 100 & 100 & 100 & 100 & 100 \\
\hline $\begin{array}{l}\text { Resistance to ethambutol } \\
(5 \mu \mathrm{g} / \mathrm{ml})\end{array}$ & 0 & 0 & 84 & 0 & 100 & 0 & 10 & 0 & 20 \\
\hline Tolerance to $0.1 \%$ nitrite & 0 & 0 & 0 & 0 & 0 & 0 & 0 & 0 & 100 \\
\hline $\begin{array}{l}\text { Tolerance to } 0.2 \% \text { picric } \\
\text { acid }\end{array}$ & 0 & 0 & 0 & 0 & 0 & 0 & 0 & 0 & 100 \\
\hline
\end{tabular}

${ }^{a} M$. tuberculosis and the $M$. bovis-M. africanum-M. microti complex are differentiated by resistance to TCH (thiopene-2-carboxylic acid hydrazide), nitrate reduction, and the 2-week arylsulfatase test. These two subgroups form the TB complex. Only species for which more than four strains were studied are shown in the table. 


\begin{tabular}{|c|c|c|c|c|c|c|c|}
\hline \multirow[b]{2}{*}{ Character } & \multicolumn{7}{|c|}{$\%$ of strains showing positive reaction } \\
\hline & 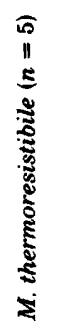 & 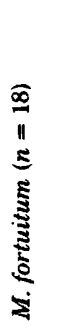 & 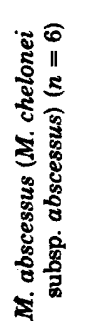 & 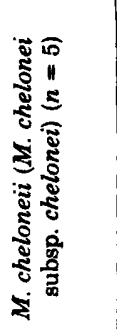 & 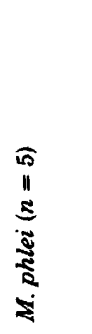 & 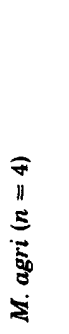 & 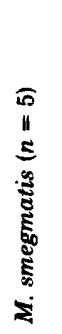 \\
\hline Pigmentation of colonies & 100 & 0 & 0 & 0 & 100 & 0 & 0 \\
\hline $\begin{array}{l}\text { Tolerance to } 0.2 \% \text { picric acid } \\
\text { (Sauton agar) }\end{array}$ & 100 & 100 & 100 & 0 & 100 & 100 & 100 \\
\hline $\begin{array}{l}\text { Resistance to } \mathrm{NH}_{2} \mathrm{OH}-\mathrm{HCl} \\
(0.25 \mathrm{mg} / \mathrm{ml})\end{array}$ & 0 & 100 & 100 & 100 & 0 & 0 & 60 \\
\hline Tween hydrolysis (7 days) & 100 & 0 & 0 & 0 & 100 & 100 & 100 \\
\hline$\alpha$-Esterase & 100 & 0 & 0 & 100 & 100 & 100 & 0 \\
\hline$\beta$-Esterase & 100 & 100 & 100 & 100 & 100 & 100 & 0 \\
\hline Acid phosphatase & 100 & 100 & 17 & 100 & 100 & 0 & 0 \\
\hline$\beta$-Galactosidase & 0 & 6 & 100 & 100 & 100 & 100 & 100 \\
\hline Nitrate reduction ( $24 \mathrm{~h}$ ) & 100 & 100 & 0 & 0 & 100 & 100 & 100 \\
\hline Arylsulfatase ( 3 days) & 0 & 100 & 100 & 100 & 0 & 0 & 0 \\
\hline Arylsulfatase (2 weeks) & 0 & 100 & 100 & 100 & 100 & 100 & 100 \\
\hline Salicylate degradation & 0 & 89 & 100 & $\mathbf{5 0}$ & 0 & 0 & 0 \\
\hline $\begin{array}{l}\text { p-Aminosalicylic acid degra- } \\
\text { dation }\end{array}$ & 0 & 89 & 100 & 100 & $\mathbf{0}$ & $\mathbf{0}$ & 0 \\
\hline Acetamidase & 0 & 100 & $\mathbf{0}$ & 100 & 0 & 100 & 100 \\
\hline Benzamidase & 0 & 0 & 0 & 0 & $\mathbf{0}$ & 0 & 100 \\
\hline Isonicotinamidase & 0 & 0 & 0 & 0 & 0 & 0 & 100 \\
\hline Allantoinase & 0 & 100 & 0 & 0 & 0 & 100 & 60 \\
\hline Growth at $45^{\circ} \mathrm{C}$ & 100 & 17 & 0 & 0 & 100 & 100 & 100 \\
\hline Growth at $52^{\circ} \mathrm{C}$ & 100 & 0 & 0 & 0 & 100 & 0 & $\mathbf{0}$ \\
\hline$n$-Propanol as C source & 100 & 100 & 0 & $\mathbf{0}$ & 100 & 100 & 100 \\
\hline$n$-Butanol as C source & 100 & 100 & $\mathbf{0}$ & 100 & 100 & 100 & 100 \\
\hline iso-Butanol as $\mathrm{C}$ source & 100 & 100 & 0 & 0 & 100 & 25 & 100 \\
\hline Propylene glycol as C source & 0 & 100 & 0 & 20 & 40 & 25 & 100 \\
\hline Galactose as C source & 0 & 0 & 0 & 0 & 80 & 0 & 100 \\
\hline Arabinose as $\mathrm{C}$ source & 0 & 0 & 0 & 0 & 100 & 0 & 100 \\
\hline Xylose as C source & 0 & 0 & 0 & 0 & 40 & 100 & 100 \\
\hline Rhamnose as C source & 0 & 0 & 0 & 0 & 0 & 0 & 100 \\
\hline Trehalose as C source & 0 & 56 & 67 & 100 & 80 & 0 & 100 \\
\hline Inositol as $\mathrm{C}$ source & 0 & 0 & 0 & 0 & 0 & 100 & 100 \\
\hline Mannitol as C source & $\mathbf{0}$ & 56 & 0 & 0 & 100 & 0 & 100 \\
\hline Sorbitol as $\mathrm{C}$ source & $\mathbf{0}$ & 0 & 0 & 0 & 100 & 0 & 100 \\
\hline Citrate as $\mathrm{C}$ source & 0 & 44 & 0 & 100 & 0 & 0 & 100 \\
\hline Succinate as $\mathrm{C}$ source & 0 & 100 & 100 & 20 & 100 & 100 & 100 \\
\hline Malate as C source & 80 & 100 & 100 & 0 & 100 & 100 & 100 \\
\hline Benzoate as $\mathrm{C}$ source & 0 & 0 & 0 & 0 & 0 & 0 & 100 \\
\hline Malonate as $\mathrm{C}$ source & 0 & 0 & 0 & 0 & 100 & 0 & 100 \\
\hline Serine as $\mathbf{N}$ and $\mathrm{C}$ source & 0 & 44 & 100 & 100 & 0 & $\mathbf{0}$ & 100 \\
\hline $\begin{array}{l}\text { Glucosamine as } \mathbf{N} \text { and } \mathbf{C} \\
\text { source }\end{array}$ & 0 & 100 & 33 & 0 & 20 & 100 & 100 \\
\hline Acetamide as $\mathrm{N}$ and $\mathrm{C}$ source & 0 & 61 & 0 & 0 & 100 & 0 & 160 \\
\hline Benzamide as $\mathrm{N}$ and $\mathrm{C}$ source & 0 & 0 & 0 & 0 & 0 & 0 & 100 \\
\hline $\begin{array}{l}\text { Monoethanolamine as } \mathrm{N} \text { and } \\
\mathrm{C} \text { source }\end{array}$ & 0 & 100 & 100 & 100 & 20 & 0 & 100 \\
\hline $\begin{array}{l}\text { Trimethylenediamine as } \mathrm{N} \\
\text { and } \mathrm{C} \text { source }\end{array}$ & 0 & 100 & 100 & 0 & 100 & 0 & 100 \\
\hline Nitrite as $\mathbf{N}$ source & 0 & 89 & 100 & 0 & 100 & $\mathbf{0}$ & 100 \\
\hline $\begin{array}{l}\text { Resistance to ethambutol (5 } \\
\mu \mathrm{g} / \mathrm{ml} \text { ) }\end{array}$ & 20 & 100 & 100 & 100 & 100 & $\mathbf{0}$ & 100 \\
\hline
\end{tabular}

${ }^{a}$ Only species for which more than four strains were studied are shown in the table. Tests for the utilization of sole carbon sources included ammoniacal nitrogen in the medium. 
TABLE 5. Distinguishing characters for species of cluster $D^{a}$

\begin{tabular}{|c|c|c|c|c|c|c|c|c|c|c|c|c|}
\hline \multirow[b]{2}{*}{ Character } & \multicolumn{12}{|c|}{$\%$ of strains showing positive reaction } \\
\hline & 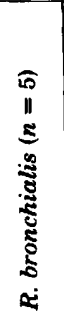 & 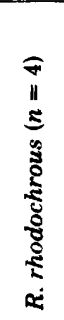 & 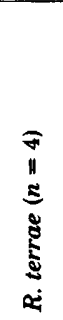 & 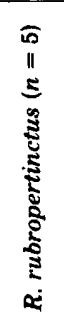 & 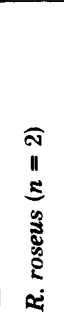 & 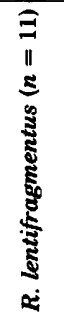 & 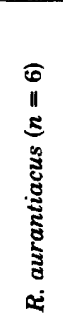 & 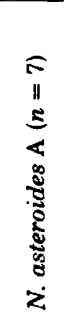 & 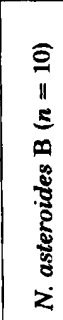 & 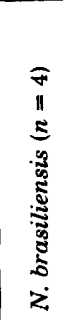 & 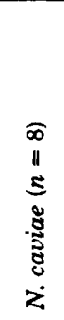 & 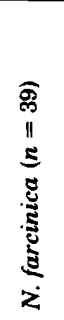 \\
\hline Fragmenting mycelium & $\mathbf{0}$ & 25 & 0 & 0 & 0 & 82 & $\mathbf{0}$ & 100 & 100 & 100 & 100 & 100 \\
\hline $\begin{array}{l}\text { Resistance to rifampin } \\
(25 \mu \mathrm{g} / \mathrm{ml})\end{array}$ & 100 & 100 & 100 & 100 & 100 & 0 & 100 & 86 & 90 & 100 & 100 & 97 \\
\hline $\begin{array}{l}\text { Tween hydrolysis ( } 7 \\
\text { days) }\end{array}$ & 0 & 0 & 0 & 0 & 0 & 0 & 83 & 0 & 0 & 0 & 0 & 0 \\
\hline $\begin{array}{l}\text { Tween hydrolysis (14 } \\
\text { days) }\end{array}$ & 0 & 0 & 0 & 0 & 0 & 0 & 100 & 43 & 20 & 0 & 75 & 64 \\
\hline$\alpha$-Esterase & 0 & 0 & 100 & 0 & 0 & 0 & 0 & 0 & 100 & 75 & 0 & 5 \\
\hline$\beta$-Esterase & 100 & 100 & 100 & 100 & 100 & $\mathbf{0}$ & 100 & 29 & 100 & 100 & 62 & 79 \\
\hline Acid phosphatase & 100 & 0 & 0 & 0 & 0 & $\mathbf{0}$ & 0 & 43 & 0 & 75 & 75 & $\mathbf{5}$ \\
\hline $\begin{array}{l}\text { Catalase (semiquantita- } \\
\text { tive) }\end{array}$ & 100 & 100 & 100 & 0 & 50 & 73 & 17 & 6 & 40 & 75 & 75 & 28 \\
\hline$\beta$-Galactosidase & 0 & 0 & 0 & 0 & 0 & 0 & 100 & 100 & 100 & 100 & 100 & 100 \\
\hline Nitrate reduction & 100 & 100 & 100 & 100 & 100 & 91 & 0 & 14 & 70 & 0 & $\mathbf{0}$ & 3 \\
\hline Acetamidase & 100 & 50 & 0 & 0 & 100 & 100 & 100 & 0 & 30 & 25 & 0 & 100 \\
\hline Benzamidase & 0 & 0 & 0 & $\mathbf{0}$ & 0 & 0 & 17 & 0 & $\mathbf{0}$ & 0 & 0 & 69 \\
\hline Urease & 100 & 25 & 100 & 100 & 100 & 18 & 100 & 100 & 70 & 100 & 100 & 100 \\
\hline Nicotinamidase & 100 & 0 & 100 & 20 & 0 & 82 & 100 & 57 & 80 & 50 & 100 & 95 \\
\hline Pyrazinamidase & 100 & 0 & 100 & 20 & 0 & 82 & 100 & 43 & 60 & 25 & 88 & 95 \\
\hline Allantoinase & 0 & 0 & 100 & 100 & 0 & 18 & 100 & 57 & 20 & 75 & 88 & 10 \\
\hline Growth at $45^{\circ} \mathrm{C}$ & 0 & 0 & 0 & 0 & 0 & 0 & 0 & $\mathbf{0}$ & 0 & 0 & 0 & 82 \\
\hline Sucrose as $\mathrm{C}$ source & 100 & 100 & 100 & 100 & 100 & 45 & 100 & 0 & 0 & $\mathbf{0}$ & 13 & 26 \\
\hline $\begin{array}{l}\text { Propylene glycol as C } \\
\text { source }\end{array}$ & 100 & 100 & 100 & 100 & 100 & 100 & 100 & 0 & 0 & 0 & 0 & 64 \\
\hline Galactose as C source & $\mathbf{0}$ & $\mathbf{0}$ & 0 & 0 & 0 & $\mathbf{0}$ & 100 & 0 & 0 & 100 & 0 & 3 \\
\hline Rhamnose as C source & $\mathbf{0}$ & 0 & 100 & $\mathbf{0}$ & $\mathbf{0}$ & $\mathbf{0}$ & $\mathbf{0}$ & $\mathbf{0}$ & 0 & 0 & $\mathbf{0}$ & 85 \\
\hline Trehalose as C source & 100 & 0 & 100 & 100 & 100 & $\mathbf{0}$ & 100 & 0 & 0 & 100 & 25 & 26 \\
\hline Inositol as C source & 100 & $\mathbf{0}$ & 0 & $\mathbf{0}$ & $\mathbf{0}$ & $\mathbf{0}$ & 100 & 0 & $\mathbf{0}$ & 100 & 75 & 3 \\
\hline Mannitol as C source & 0 & 0 & 100 & 100 & 100 & 100 & 100 & $\mathbf{0}$ & 0 & 75 & 100 & 3 \\
\hline Sorbitol as C source & $\mathbf{0}$ & 0 & 100 & 100 & 100 & 91 & 100 & 100 & $\mathbf{0}$ & $\mathbf{0}$ & $\mathbf{0}$ & 0 \\
\hline Citrate as C source & 20 & 50 & 50 & 100 & 100 & 100 & 100 & 86 & 0 & 100 & 13 & $\mathbf{5}$ \\
\hline Benzoate as $\mathrm{C}$ source & $\mathbf{0}$ & 75 & $\mathbf{0}$ & 100 & 50 & 100 & 33 & $\mathbf{0}$ & $\mathbf{0}$ & $\mathbf{0}$ & 0 & $\mathbf{0}$ \\
\hline Serine as $\mathrm{N}$ and $\mathrm{C}$ source & $\mathbf{0}$ & 0 & 0 & 0 & 0 & 9 & 100 & 0 & 0 & $\mathbf{0}$ & 100 & 8 \\
\hline $\begin{array}{l}\text { Glucosamine as } \mathrm{N} \text { and } \mathrm{C} \\
\text { source }\end{array}$ & 20 & 100 & 50 & 100 & 100 & 82 & 100 & 57 & $\mathbf{0}$ & 75 & 100 & 95 \\
\hline $\begin{array}{l}\text { Acetamide as } \mathrm{N} \text { and } \mathrm{C} \\
\text { source }\end{array}$ & 100 & $\mathbf{0}$ & $\mathbf{0}$ & $\mathbf{0}$ & 100 & $\mathbf{0}$ & 100 & 0 & $\mathbf{0}$ & 0 & 0 & 95 \\
\hline $\begin{array}{l}\text { Monoethanolamine as } \mathrm{N} \\
\text { and C source }\end{array}$ & $\mathbf{0}$ & 0 & 75 & 100 & $\mathbf{0}$ & 91 & 100 & $\mathbf{0}$ & 0 & $\mathbf{0}$ & 88 & 100 \\
\hline Benzamide as $\mathbf{N}$ source & 0 & $\mathbf{0}$ & 75 & 40 & $\mathbf{0}$ & $\mathbf{0}$ & $\mathbf{0}$ & $\mathbf{0}$ & 70 & 0 & 0 & 69 \\
\hline
\end{tabular}

a Tests for the utilization of sole carbon sources included ammoniacal nitrogen in the medium.

mycobacteria and rapidly growing mycobacteria be placed in taxa comparable to Nocardia or Gordona (now Rhodococcus). This suggestion was supported by the results obtained from the present study.

Cluster $\mathrm{D}$ was divided into approximately two subclusters, one composed of the strains of Rho- dococcus and another consisting of the strains of Nocardia. $R$. aurantiacus and J. canicruria (according to Goodfellow and Alderson [3], $J$. canicruria is $R$. erythropolis) were located in cluster D but were located on the outside of these subclusters. The single strain of $C$. equi studied was incorporated into cluster $D$. This 


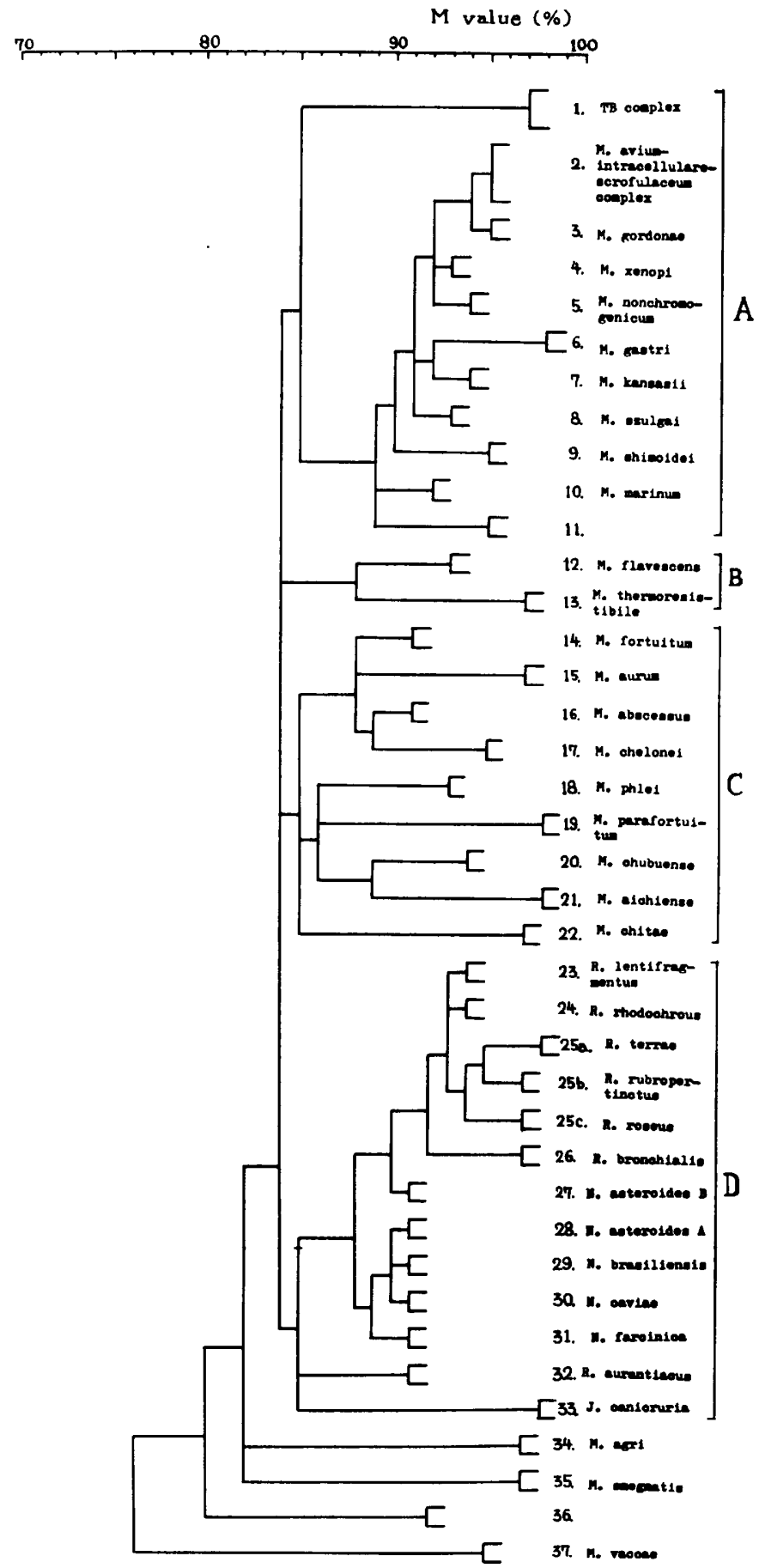

Fig. 7. General view of dendrogram. Cluster A, Subclusters 1 to 11 ; cluster B, subclusters 12 and 13; cluster C, subclusters 14 to 22 ; cluster $D$, subclusters 23 to 33 . 
appears to be reasonable, because $C$. equi was recently transferred by Goodfellow and Alderson (3) to Rhodococcus as $R$. equi.

$N$. asteroides was divided into two subclusters, 27 and 28. Previously, $N$. asteroides was divided by Tsukamura (15) into two species, $N$. asteroides in the strict sense and $N$. farcinica. The former was recently further divided into two subgroups (23). The same findings were obtained in the present study. The strains received as $N$. asteroides were divided into three subgroups, $N$. asteroides $\mathrm{A}, \boldsymbol{N}$. asteroides $\mathrm{B}$, and $N$. farcinica. After the study of Tsukamura (15), Goodfellow (2), Kurup and Schmidt (7), and Mordarski et al. (9) divided $N$. asteroides into five, four, and three subgroups, respectively. One of the subgroups of Mordarski et al. (9) is probably identical to $N$. farcinica of Tsukamura (15). The determination of the relationships of the subgroups of these authors remains a problem for future study.

As shown previously by Tsukamura $(14,17$, 21) and in the present study, slowly growing and rapidly growing mycobacteria seem to be valid taxa of generic (or subgeneric) rank. Strains of a few species of rapidly growing mycobacteria remained as outsiders, namely, those of $M$. agri, $M$. smegmatis, and $M$. vaccae, as did strains of scotochromogenic, rapidly growing mycobacteria isolated from a bovine thelitis lesion by Shimizu et al. (11).

The strains received as members of Gordona and those designated "M." rhodochrous were regarded as belonging to a single genus, Rhodococcus, in numerical taxonomy studies carried out by Tsukamura (20) and by Goodfellow and Alderson (3), although some doubts about this conclusion have been raised $(1,6,8,10)$. The results of the present study support the previously proposed concept that the taxa heretofore designated as Gordona, "M." rhodochrous complex, and Nocardia rubra belong to one and the same taxon. It was also shown that the genera Rhodococcus and Nocardia are more closely related to each other than they are to the mycobacteria.

In a previous numerical taxonomy study by Tsukamura (22), three species, $M$. avium, $M$. intracellulare, and $M$. scrofulaceum were combined into one, the correct name of which is $M$. avium. This finding was supported in the present study.

Strains of $M$. thermoresistible and those of $M$. flavescens formed a unique cluster, $\mathrm{B}$, which is distinct from the clusters of both the slowly growing and the rapidly growing mycobacteria. In a previous study, in which some linked characters were used, $\boldsymbol{M}$. thermoresistible was placed in the cluster of slowly growing mycobacteria
(14), and in another study, in which hypothetical median organisms were used, $\boldsymbol{M}$. flavescens and $M$. thermoresistible were placed in the cluster of rapidly growing mycobacteria (21). The results obtained in the present study, in which a large number of strains were used and linked characters were omitted as far as possible, are considered to be more reliable than those of the two studies just mentioned.

\section{ACKNOWLEDGMENTS}

We express our appreciation to $H$. $H$. Kleeberg, Tuberculosis Research Institute, Pretoria, South Africa, for kind permission for the use in this study of the strains bearing the symbol TC (Table 1).

\section{REPRINT REQUESTS}

Address reprint requests to: Dr. M. Tsukamura, National Chubu Hospital, Obu, Aichi 474, Japan.

\section{LTTERATURE CITED}

1. Alshamaony, L., M. Goodfellow, D. E. Minnikin, and H. Mordarska. 1976. Free mycolic acids as criteria in the classification of Gordona and the 'rhodochrous' complex. J. Gen. Microbiol. 92:183-187.

2. Goodfellow, M. 1971. Numerical taxonomy of some nocardioform bacteria. J. Gen. Microbiol. 69:33-88.

3. Goodfellow, M., and G. Alderson. 1977. The Actinomycete-genus Rhodococcus: a home for the 'rhodochrous' complex. J. Gen. Microbiol. 100:99-122.

4. Goodfellow, M., A. Fleming, and M. J. Sackin. 1972. Numerical classification of "Mycobacterium" rhodochrous and Runyon's group IV mycobacteria. Int. J. Syst. Bacteriol. 22:81-98.

5. Goodfellow, M., A. Lind, H. Mordarska, S. Pattyn, and $M$. Trukamura. 1974. A co-operative numerical analysis of cultures considered to belong to the "rhodochrous' taxon. J. Gen. Microbiol. 85:291-302.

6. Hyman, I. S., and S. D. Chaparas. 1977. A comparative study of the 'rhodochrous' complex and related taxa by delayed-type skin reactions on guinea pigs and by polyacrylamide gel electrophoresis. J. Gen. Microbiol. 100: 363-371.

7. Kurup, P. V., and J. A. Schmidt. 1973. Numerical taxonomy of Nocardia. Can. J. Microbiol. 19:10351048.

8. Minnikin, D. E., P. V. Patel, L. Alshamaony, and M. Goodfellow. 1977. Polar lipid composition in the classification of Nocardia and related bacteria. Int. J. Syst. Bacteriol. 27:104-117.

9. Mordarski, M., K. P. Schaal, K. Szyba, G. Pulverer, and A. Tkacz. 1977. Interrelation of Nocardia asteroides and related taxa as indicated by deoxyribonucleic acid reassociation. Int. J. Syst. Bacteriol. 27:66-70.

10. Mordarski, M., K. Szyba, G. Pulverer, and M. Goodfellow. 1976. Deoxyribonucleic acid reassociation in the classification of the 'rhodochrous' complex and allied taxa. J. Gen. Microbiol. 94:235-245.

11. Shimizu, K., T. Hirose, M. Sato, and M. Tsukamura. 1977. Isolation of acid-fast organisms resembling $M y$. cobacterium vaccae from a lesion of bovine nodular thelitis. Microbiol. Immunol. 21:469-472.

12. Sokal, R. R., and P. H. A. Sneath. 1963. Principles of numerical taxonomy, p. 1-359. W. H. Freeman, San Francisco.

13. Tsukamura, M. 1966, Adansonian classification of mycobacteria. J. Gen. Microbiol. 46:253-273.

14. Tsukamura, M. 1967. Identification of mycobacteria. Tubercle 48:311-338.

15. Tsukamura, M. 1969. Numerical taxonomy of the genus 
Nocardia. J. Gen. Microbiol. 56:265-287.

16. Tsukamura, M. 1970. Differentiation between Mycobacterium abscessus and Mycobacterium borstelense. Am. Rev. Respir. Dis. 101:426-428.

17. Tsukamura, M. 1970. Relationship between Mycobacterium and Nocardia. Jpn. J. Microbiol. 14:187-195.

18. Tsukamura, M. 1971. Proposal of a new genus, Gordona, for slightly acid-fast organisms occurring in sputa of patients with pulmonary disease and in soil. J. Gen. Microbiol. 68:15-26.

19. Tsukamura, M. 1973. A toxonomic study of strains received as "Mycobacterium" rhodochrous. Description of Gordona rhodochroa (Zopf; Overbeck; Gordon et Mihm) Tsukamura comb. nov. Jpn. J. Microbiol. 17: 189-197.

20. Tsukamura, M. 1974. A further numerical taxonomic study of the Rhodochrous group. Jpn. J. Microbiol. 18: $37-44$.

21. Tsukamura, M. 1975. Numerical analysis of the relation- ship between Mycobacterium, Rhodochrous group, and Nocardia by use of hypothetical median organisms. Int. J. Syst. Bacteriol. 25:329-335.

22. Tsukamura, M. 1976. Numerical classification of slowly growing mycobacteria. Int. J. Syst. Bacteriol 26:409420.

23. Tsukamura, M. 1977. Extended numerical taxonomy study of Nocardia. Int. J. Syst. Bacteriol. 27:311-323.

24. Tsukamura, M., and S. Mizuno. 1968. "Hypothetical mean organisms" of mycobacteria. A study of classification of mycobacteria. Jpn. J. Microbiol. 12:371-384.

25. Tsukamura, M., and S. Mizuno. 1971. A new species of Gordona aurantiaca occurring in sputa of patients with pulmonary disease. Kekkaku 46:93-98.

26. Tsukamura, M., S. Mizuno, and H. Murata. 1975. Numerical taxonomy study of the taxonomic position of Nocardia rubra reclassified as Gordona lentifragmenta Tsukamura nom. nov. Int. J. Syst. Bacteriol. 25: 377-382. 